\title{
An Aloha Protocol for Multihop Mobile Wireless Networks
}

\author{
François Baccelli, Bartłomiej Błaszczyszyn, and Paul Mühlethaler
}

\begin{abstract}
An Aloha-type access control mechanism for large mobile, multihop, wireless networks is defined and analyzed. This access scheme is designed for the multihop context, where it is important to find a compromise between the spatial density of communications and the range of each transmission. More precisely, the analysis aims at optimizing the product of the number of simultaneously successful transmissions per unit of space (spatial reuse) by the average range of each transmission. The optimization is obtained via an averaging over all Poisson configurations for the location of interfering mobiles, where an exact evaluation of signal over noise ratio is possible. The main mathematical tools stem from stochastic geometry and are spatial versions of the so-called additive and max shot noise processes. The resulting medium access control (MAC) protocol exhibits some interesting properties. First, it can be implemented in a decentralized way provided some local geographic information is available to the mobiles. In addition, its transport capacity is proportional to the square root of the density of mobiles which is the upper bound of Gupta and Kumar. Finally, this protocol is self-adapting to the node density and it does not require prior knowledge of this density.
\end{abstract}

Index Terms-Medium access control (MAC) layer, multipleaccess protocol, network design, optimization, point process, queuing theory, signal-to-interference ratio, stochastic geometry, stochastic process, transport capacity.

\section{INTRODUCTION}

$\mathbf{T}$ HIS paper concentrates on the medium access control (MAC) of wireless networks with several mobile transmitters and receivers sharing a common Hertzian medium, like, for example, in certain classes of mobile ad hoc networks or sensor networks. One of the main difficulties for tuning MAC within this context stems from the mobility and the resulting unpredictability of the geometrical properties of the emission patterns. Mobility in particular may lead to random spatial clustering rendering some sets of simultaneous transmissions impossible due to high interference.

Within this context, the MAC protocols aim at defining policies where mobiles access the shared medium in such a way that spatial or temporal clustering does not occur or only rarely happens. This is done by some exclusion mechanism that prevents

Manuscript received February 13, 2004; revised May 24, 2005. The work of B. Błaszczyszyn is supported in part by Komitet Badań Naukowych (KBN), Poland, under Grant 2 P03A 02023.

F. Baccelli is with the ENS-INRIA 75230 Paris, France (e-mail: Francois. Baccelli@ens.fr).

B. Błaszczyszyn is with the ENS-INRIA, 75230 Paris, France, and with the Mathematical Institute, University of Wrocław, Wrocław, Poland (e-mail: Bartek.Blaszczyszyn@ens.fr).

P. Mühlethaler is with the INRIA HiPERCOM, Rocquencourt B.P. 105, 78153 Le Chesnay, France (e-mail: Paul.Muhlethaler@inria.fr).

Communicated by G. Sasaki, Associate Editor for Communication Networks.

Digital Object Identifier 10.1109/TIT.2005.862098 mobiles that are close to some transmitting mobile (and also receiving mobile in the case of IEEE 802.11 with the RTS-CTS option) from transmitting at the same time. In wireless networks, the MAC algorithm is supposed to prevent simultaneous neighboring transmissions from occurring, as often as possible, since such transmissions are bound to produce collisions. On the other hand, MAC protocols should allow as many simultaneous and successful transmissions as possible over different parts of the network. This ability of mobile wireless networks is known as spatial reuse.

Aloha [1] along with time-division multiple access (TDMA) was one of the first protocols used in radio networks. The work presented in this paper is an adaptation and an optimization of Aloha in the context of a multihop network of mobile nodes. This optimization of Aloha uses various mathematical tools mainly borrowed from stochastic geometry.

The paper is organized as follows. Section II reviews related work and positions the contributions of the present paper in this context.

Section III introduces the mathematical model. Section IV focuses on spatial reuse Aloha (SR-Aloha), namely, on the optimization of the medium access probability (MAP) $p$ when each station tries to make a hop of length $R$, or on the best hop length $R$ when $p$ is fixed. We show that this simple optimization fails to determine the optimal MAC setting.

The main result of the paper is introduced in Section V, where the optimization of the multihop version of SR-Aloha (MSRAloha) is addressed. We introduce a new notion, the spatial density of progress, which is a space averaging of how much packets progress toward their destinations. We show that there exists a value of the MAP which maximizes this spatial density of progress and we give an analytical characterization of the optimal MAP $p^{*}$

In Section VI, we discuss capacity and stability issues for MSR-Aloha. We consider the protocol under the following assumptions:

- each mobile initiates a stationary flow of packets of intensity $\tau$ to be transported to some random destination;

- the origin-destination (o-d) pairs are isotropic and the distance between each $\mathrm{o}-\mathrm{d}$ pair is a random variable with finite mean;

- each mobile moves according to some way point model;

- the network is in charge of transmitting packets via several hops.

A snapshot of the network at any given time consists of stations having to transmit packets in some direction and attempting to do so in a minimal number of hops, or equivalently via a successful jump as far as possible in the desired direction. We show 
that if $\tau$ is less than a threshold that is given in closed form, then MSR-Aloha is rate-stable (see Appendix A for a definition).

We comment also on the particular case where nodes have no mobility at all. In this case, the previous rate-stability result no longer holds. The protocol nevertheless provides a positive throughput to any node of the network and is still optimal in some sense.

Implementation issues are briefly discussed in Section VIII, with a particular emphasis on a decentralized implementation of the protocol. The Appendix gathers proofs of two technical results concerning the Poisson shot-noise process that are used in the paper. It also contains a comparison of SR-Aloha with the carrier sense multiple access (CSMA) technique which is the basis of the MAC protocol for the wireless local-area network's (WLAN's) standards IEEE 802.11 [2] and Hiperlan type 1 [3].

\section{State of the ART AND Related Works}

Aloha is a widely deployed and studied access protocol. The initial paper presenting Aloha was published in 1970 [1] and Aloha is now used in most cellular networks to request access. A lot of both theoretical and practical studies have been carried out to improve Aloha. Initial studies [4], [5] looked for methods to stabilize the protocol. The first paper studying Aloha in a multihop context was [6]. In this work, Nelson and Kleinrock computed the probability of successful transmission in a random planar Aloha packet radio network with a simple model where interferences only propagate two hops away. In 1988, Ghez, Verdú, and Schwartz introduced a model for slotted Aloha with multipacket reception capability in a widely referenced paper [7], which introduced a well-accepted model for Aloha in a network with spatial reuse.

The present paper revisits the spatial reuse Aloha MAC mechanism in the context of multihop mobile wireless networks. Compared with [7], the main new contributions of the present paper are as follows.

- An exact representation of the signal-to-noise-and-interference ratio (SINR) at the receiver, for each transmission, and hence, of the collisions of the Aloha scheme, taking into account all interferers; within this context, the occurrence of a collision will be determined by the SINR at the receiver, which is justified under the classical Gaussian channel model of information theory.

- Various optimizations of the Aloha protocol: the optimization for SR-Aloha concerns the case where some predefined transmission range is set; that for MSR-Aloha meant for the multihop context and which aims to transmit a packet "as far as possible" while taking into account the reception probability. The routing protocol that will be considered here is close to the most forward with radius (MFR). In this greedy routing protocol introduced by Takagi and Kleinrock [8], a node selects the neighbor with the shortest projected distance to the receiver. The main new step of the present paper concerning multihop optimization is the merging of the geometric routing notion of "most forwarding" with the MAC notion of "transmission success," which is also of a geometric nature because of SINR, into a unique geometric function to optimize.
Keeping the "random access" spirit of the Aloha protocol, numerous studies tried to design more efficient protocols. Two main approaches have been investigated; the first one consists of taking advantage of the history of the channel in order to adopt a better retransmission strategy than the blind Aloha re-emission strategy. The second one consists of improving the control of the channel by carrier sensing: that is, the carrier sense multiple access (CSMA) technique. In [9], it is shown that CSMA actually outperforms Aloha in wired networks. However, Tobagi points out in [10] that CSMA protocols may suffer from hidden collisions and numerous papers, mostly in the 1990s, proposed dedicated protocols to cope with this problem [11]-[14]. Actually, these protocols can be seen as enhanced CSMA protocols where the carrier sense effect is also used around the receiver to protect its reception. As a byproduct of the proposed analytical model, the present paper offers a tentative comparison between Aloha and CSMA in a multihop network under the general SINR model (see the Appendix, subsection B ). Following the above remark, this is a pertinent comparison since we may question the benefit of CSMA in an ad hoc network if we have to suffer from hidden nodes.

In 2000, Gupta and Kumar published a now widely referenced article [15] in which they show how the throughput of a multihop ad hoc network scales with node density. This analysis is made for various communication channel models including the SINR model (used in the present article), and for various user location models. The random location model of [15] consists of $\lambda$ nodes uniformly distributed in a unit area. The analysis is based on a random traffic pattern where each node chooses at random a destination in the unit area. If the lower bound of [15] is used for this random location-SINR model, we find that an $a d$ hoc network using a CSMA scheme with an appropriate carrier sense range can sustain a total transport capacity of the order of $O\left(\frac{\sqrt{\lambda}}{\sqrt{\log (\lambda)}}\right)$. Gupta and Kumar give access and routing protocols allowing the network to reach this bound; however, the proposed solution does not allow to derive a distributed protocol allowing us to reach the estimate.

Although the present paper also uses the SINR model, its location model differs from that of [15] in that we take an infinite plane where users are randomly located according to a Poisson point process of intensity $\lambda$ rather than a finite domain where the number of users increases to infinity. The traffic pattern is similar to that of [15] in that each node chooses a random destination at some finite distance. The relationships between the two models will be discussed further in Section VI.

We will show that MSR-Aloha is an implementable access scheme that gives a density of progress (a notion related to Gupta and Kumar's transport capacity) of the form $K(p) \sqrt{\lambda}$, where $p$ is the MAP; we will also give a closed form for $K(p)$, allowing for an optimization with respect to $p$, which is one of the main results of the paper. The obtained order $O(\sqrt{\lambda})$ is the same as the upper bound in Gupta and Kumar. We also explain why MSR-Aloha does better than the $O\left(\frac{\sqrt{\lambda}}{\sqrt{\log (\lambda)}}\right)$ scheme in Gupta and Kumar. First, this result requires some node mobility whereas the Gupta and Kumar scheme does not. Another important difference is that although it allows the transmission of packets over time and in several hops from any node to any 
other node, MSR-Aloha does not require any connectivity at a given time whereas the Gupta and Kumar scheme does. In ad hoc networks, connectivity is usually enforced via complex neighborhood management algorithms which lead to significant overhead (see, e.g., [16]-[19]). The fact that connectivity is not required by MSR-Aloha can thus be thought of as an important additional argument in favor of this access protocol.

\section{A Stochastic Geometry Model For SPATIAL Reuse AlOHA}

We consider an infinite planar network. Let

$$
\Phi=\left\{\left(X_{i},\left(e_{i}, S_{i}, T_{i}\right)\right)\right\}
$$

be a marked Poisson point process with intensity $\lambda$ on the plane $\mathbb{R}^{2}$, where we have the following.

- $\Phi=\left\{X_{i}\right\}$ denotes the locations of the stations;

- $\left\{e_{i}\right\}_{i}$ the medium access indicator of station $i ; e_{i}=1$ for the stations which are allowed to transmit, and $e_{i}=0$ means the station is (a potential) receiver. Here, the random variables $e_{i}$ are independent, with $\boldsymbol{P}\left(e_{i}=1\right)=p$.

- $\left\{S_{i}\right\}_{i}$ denotes the powers emitted by the stations (stations for which $e_{i}=1$ ); the random variables $\left\{S_{i}\right\}$ will always be assumed independent and identically distributed (i.i.d.) with mean $1 / \mu$. Unless otherwise specified, the $S_{i}$ 's have a general distribution. Under this general distribution assumption, we will be able to prove our qualitative results. An important special case, in which a quantitative analysis is possible, is that with exponentially distributed powers. ${ }^{1}$

- $\left\{T_{i}\right\}$ are the SINR thresholds corresponding to some channel bit rates or bit-error rates; here, for simplicity, we will take $T_{i} \equiv T$ constant.

In addition to this marked point process, the model is based on a function $l(x, y)$ that gives the attenuation (path loss) from $y$ to $x$ in $\mathbb{R}^{2}$. We will assume that the path loss depends only on the distance, i.e, with a slight abuse of notation $l(x, y)=$ $l(|x-y|)$. As an important special case of the simplified attenuation function we will take

$$
l(u)=(A u)^{-\beta}, \quad \text { for } A>0 \text { and } \beta>2 .
$$

Note that such $l(u)$ explodes at $u=0$, and thus in particular is not correct for a small distance $r$ and large intensities $\lambda$; cf Remark 4.4.

We also consider an independent external noise (i.e., independent of $\Phi$, e.g., thermal) and denote it (at a given location) by $W$.

Note first that $\Phi$ can be represented as a pair of independent Poisson point processes representing transmitters $\Phi^{1}=\left\{X_{i}\right.$ : $\left.e_{i}=1\right\}$ and receivers $\Phi^{0}=\left\{X_{i}: e_{i}=0\right\}$, with intensities, respectively, $\lambda p$ and $\lambda(1-p)$.

Let us suppose there is a station located at $x$ that transmits with power $S$ and requires SINR $T$. Suppose there is a user

\footnotetext{
${ }^{1}$ Note also that a constant emitted power combined with Rayleigh fading leads to a random exponential received power.
}

located at $y \in \mathbb{R}^{2}$. The station can establish a channel to this user with a given bit rate (which will be taken as the unit throughput in what follows) if and only if

$$
\frac{S l(|x-y|)}{W+I_{\Phi^{1}}(y)} \geq T
$$

where $I_{\Phi^{1}}$ is the shot-noise process of $\Phi^{1}: I_{\Phi^{1}}(y)=$ $\sum_{X_{i} \in \Phi^{1}} S_{i} l\left(\left|y-X_{i}\right|\right)$. Denote by $\delta\left(x, y, \Phi^{1}\right)$ the indicator that (3.2) holds. Note that by stationarity of $\Phi^{1}$, the probability $E\left[\delta\left(x, y, \Phi^{1}\right)\right]$ depends only on the distance $x-y$ and not on the specific locations of $(x, y)$; so we can use the notation

$$
p_{|x-y|}(\lambda p)=\boldsymbol{E}\left[\delta\left(x, y, \Phi^{1}\right)\right]
$$

where $\lambda p$ is the intensity of the transmitters $\Phi^{1}$. The following lemma is the basis of our quantitative analysis of the model.

Lemma 3.1: For exponential $S$ with mean $1 / \mu$

$$
p_{R}(\lambda)=\exp \left\{-2 \pi \lambda \int_{0}^{\infty} \frac{u}{1+l(R) /(T l(u))} \mathrm{d} u\right\}_{\psi_{W}(\mu T / l(R))}
$$

where $\psi_{W}(\cdot)$ is the Laplace transform of $W$.

Proof: The proof is based on additive shot noise theory. Note by (3.2) that

$$
\begin{aligned}
p_{R}(\lambda) & =P\left(S \geq T\left(W+I_{\Phi}\right) / l(R)\right) \\
& =\int_{0}^{\infty} e^{-\mu s T / l(R)} \mathrm{d} \operatorname{Pr}\left(W+I_{\Phi} \leq s\right) \\
& =\psi_{I_{\phi}}(\mu T / l(R)) \psi_{W}(\mu T / l(R))
\end{aligned}
$$

where $\psi_{I_{\Phi}}(\cdot)$ is the Laplace transform of $I_{\Phi}=I_{\Phi}(0)$. Note that $\psi_{W}$ does not depend on $\lambda$. It is known that for a general Poisson shot noise

$$
\psi_{I_{\Phi}}(\xi)=\exp \left\{-\lambda \int_{\mathbb{R}^{2}} 1-\boldsymbol{E}\left[e^{-\xi S l(|x|)}\right] \mathrm{d} x\right\} .
$$

Since $S$ is exponential with mean $1 / \mu$, we have

$$
\begin{aligned}
\psi_{I_{\Phi}}(\xi) & =\exp \left\{-\lambda \int_{\mathbb{R}^{2}} 1-\frac{\mu}{\mu+\xi l(|x|)} \mathrm{d} x\right\} \\
& =\exp \left\{-2 \pi \lambda \int_{0}^{\infty} \frac{u}{1+\mu /(\xi l(u))} \mathrm{d} u\right\}
\end{aligned}
$$

that concludes the proof.

Corollary 3.2: For exponential $S, W \equiv 0$, and the simplified attenuation function (3.1)

$$
p_{R}(\lambda)=e^{-\lambda R^{2} T^{2 / \beta} C}
$$

where

$$
C=C(\beta)=(2 \pi \Gamma(2 / \beta) \Gamma(1-2 / \beta)) / \beta
$$

and $\Gamma(z)=\int_{0}^{\infty} t^{z-1} e^{-t} \mathrm{~d} t$ is the Gamma function.

For a general power distribution, we do not know any explicit form of $p_{R}(\lambda)$. However, the following scaling result will be 
useful when analyzing the model. Denote by $\bar{p}_{r}$ the value of $p_{r}(1)$ calculated for the model with the simplified attenuation function (3.1), $T \equiv 1, W \equiv 0$, and normalized emitted powers $\bar{S}_{i}=\mu S_{i}$.

Lemma 3.3: For a general power distribution and the simplified attenuation function (3.1), when the external noise $W \equiv 0$, we have

$$
p_{R}(\lambda)=\bar{p}_{R T^{1 / \beta} \lambda^{1 / 2}}
$$

Note that $\bar{p}_{r}$ does not depend on any parameter of the model other than the distribution of the normalized emitted power $\bar{S}$.

Proof: The Poisson point process $\Phi$ with intensity $\lambda>0$ can be represented as $\left\{X_{i}^{\prime} / \sqrt{\lambda}\right\}$, where $\Phi^{\prime}=\left\{X_{i}^{\prime}\right\}$ is Poisson with intensity 1 . Due to this, the Poisson shot noise with simplified attenuation function can take the following representation: $I_{\Phi}=\lambda^{\beta / 2} I_{\Phi^{\prime}}$. Thus, for $W=0$

$$
\begin{aligned}
p_{R}(\lambda) & =\operatorname{Pr}\left(S \geq T(A R)^{\beta} I_{\Phi}\right) \\
& =\operatorname{Pr}\left(\mu S \geq \mu\left(A R T^{1 / \beta} \lambda^{1 / 2}\right)^{\beta} I_{\Phi^{\prime}}\right) \\
& =\bar{p}_{R T^{1 / \beta} \lambda^{1 / 2}} .
\end{aligned}
$$

\section{ONE-HOP ANALYSIS}

In this section, we suppose that each mobile $X_{i} \in \Phi$ attempts to transmit to one receiver $Y_{i}$ located at a distance $R=\left|X_{i}-Y_{i}\right|$ via a channel where the success of the transmission is based on (3.2).

\section{A. SR-Aloha: Best MAP Given Some Range}

The first question that we investigate assumes that the range of all transmissions is given and looks for the value of MAP $p$ that maximizes the mean number of transmitters (and thus transmitter-receiver pairs) that can successfully transmit, per unit area. The main result is that there exists an optimal MAP and thus a way to optimize Aloha once the transmission range is fixed. The associated protocol will be referred to as SR-Aloha in what follows.

In fact, we do not ask here whether there is a receiver $Y_{i}$ located at a distance $R$ as we will do in the next section. This is why there is actually only one point process of intensity $\lambda^{1}=$ $\lambda p$ in the model in this section, and the optimization in $p$ can actually be seen as that in the variable $\lambda^{1}$. In order to simplify notation, we will drop the upper index 1 in this section, call $\Phi$ the point process of transmitters with intensity $\lambda$, and look for the optimal $\lambda$.

We have the following simple formula for the spatial density of successful transmissions in the network.

Proposition 4.1: The mean number of transmitters per unit area that can successfully transmit at range $R$ is $\lambda p_{R}(\lambda)$.

Proof: For $B \subset \mathbb{R}^{2}$ of unit area

$$
\begin{aligned}
& \boldsymbol{E}\left[\#\left\{X_{i} \in B: \delta\left(X_{i}, Y_{i}, \Phi\right)=1\right\}\right] \\
& =\boldsymbol{E}\left[\sum_{X_{i} \in \Phi} \mathbb{1}\left(X_{i} \in B\right) \delta\left(X_{i}, Y_{i}, \Phi\right)\right] \\
& =\lambda \int_{\mathbb{R}^{2}} \mathbb{1}(x \in B) p_{R}(\lambda) \mathrm{d} x=\lambda p_{R}(\lambda) .
\end{aligned}
$$

Now we look for the intensity

$$
\lambda_{\max }=\arg \max _{\lambda \geq 0}\left\{\lambda p_{R}(\lambda)\right\}
$$

that maximizes the spatial density of successful transmissions in the network. We will see that for, exponential $S, \lambda_{\max }$ is well defined. For a general $S$, we have the following technical result on the matter.

Lemma 4.2: If $\operatorname{Pr}(S>0)>0$ then $p_{r}(\lambda)$ is continuous in $\lambda$. If moreover $\boldsymbol{E}\left[S^{3 / \beta}\right]<\infty$ then $\lim _{\lambda \rightarrow \infty} \lambda p_{r}(\lambda)=0$ for any $r>0$.

The proof is given in the Appendix . As a consequence of Lemma 4.2, the function $\lambda \mapsto \lambda p_{r}(\lambda)$ attains its maximum in $(0, \infty)$. Define $\lambda_{\max }$ to be the smallest $\lambda$ for which the spatial density of successful transmissions is maximal. Then $0<$ $\lambda_{\max }<\infty$.

Proposition 4.3: For a general $S$, the simplified attenuation function (3.1) and $W \equiv 0$

$$
\begin{aligned}
\lambda_{\max } & =\frac{c_{1}}{R^{2} T^{2 / \beta}} \\
\lambda_{\max } p_{R}\left(\lambda_{\max }\right) & =\frac{c_{2}}{R^{2} T^{2 / \beta}},
\end{aligned}
$$

where the constants $c_{1}, c_{2}$ do not depend on $R, T, \mu$, provided $\lambda_{\max }$ is well defined. For exponential $S, c_{1}=1 / C$ and $c_{2}=$ $1 /(e C)$ with $C$ as defined in (3.5).

\section{Proof: Suppose}

$$
\lambda_{\max }=\arg \max _{\lambda \geq 0}\left\{\lambda p_{R}(\lambda)\right\}
$$

is well defined. By Lemma 3.3

$$
c_{1}=\arg \max _{\lambda \geq 0}\left\{\lambda \bar{p}_{\lambda^{1 / 2}}\right\}
$$

and

$$
c_{2}=\max _{\lambda \geq 0}\left\{\lambda \bar{p}_{\lambda^{1 / 2}}\right\}
$$

which completes the proof for general $S$. For exponential $S$, the general attenuation function and the general distribution of $W \geq 0$, by Lemma 3.1, and the differentiation of the function $\lambda p_{R}(\lambda)$ with respect to (w.r.t.) $\lambda$, it is easy to see that its unique maximum is attained at

$$
\lambda_{\max }=\left(2 \pi \int_{0}^{\infty} \frac{u}{1+l(R) /(T l(u))} \mathrm{d} u\right)^{-1}
$$

and the maximal value is given by

$$
\lambda_{\max } p_{R}\left(\lambda_{\max }\right)=e^{-1} \lambda_{\max } \psi_{W}(\mu T / l(R)) .
$$

Inserting the simplified attenuation function (3.1) and $W \equiv 0$ and evaluating the integral

$$
2 \pi \int_{0}^{\infty} \frac{u}{1+l(R) /(T l(u))} \mathrm{d} u=\frac{2 \pi R^{2} T^{2 / \beta}}{\beta} \Gamma(2 / \beta) \Gamma(1-2 / \beta)
$$

we get the result. 
Note that under this optimal choice of $\lambda$, the mean distance progressed by transmissions per unit space, for exponential $S$ and the simplified attenuation function, is

$$
R \lambda_{\max } p_{R}\left(\lambda_{\max }\right)=\frac{c_{2}}{R T^{2 / \beta}}
$$

which is maximal for $R=0$.

Remark 4.4: Note that in Proposition 4.3 we assumed the simplified attenuation function $l(u)=(A u)^{-\beta}$ which explodes at $u=0$, and thus in particular is not correct for a small distance $r$ and large intensities $\lambda$. There are more accurate models of short-range attenuation, e.g., $l(u)=\left(A \max \left(r_{0}, u\right)\right)^{-\beta}$ or $l(u)=(1+A u)^{-\beta}$ for some $A>0, r_{0}>0$, and $\beta>2$. Then the constants $c_{1}$ and $c_{2}$ can be evaluated numerically for exponential $S$.

\section{B. Spatial Reuse}

We can also interpret the last results in terms of the so-called spatial reuse factor defined as the distance to the receiver $R$ divided by the (mean) distance between adjacent transmitters. For this last quantity, we take the mean distance between neighboring points in the Poisson-Voronoi tessellation (more precisely, the mean edge length of the typical triangle in the Poisson-Delaunay triangulation), which is $32 /\left(9 \pi \sqrt{\lambda_{\max }}\right)$. For exponential $S$, we get Spatial reuse $=T^{-1 / \beta} \frac{9 \pi}{32 \sqrt{C}}$. For the network based on the perfect triangular mesh (i.e., network nodes are at the vertices of equilateral triangles generated by an equilateral triangle infinitely replicated by symmetry), spatial reuse was analyzed in [20] and is given by the formula

$$
T^{-1 / \beta} \frac{\sqrt{3}}{2(6 \zeta(\beta-1))^{1 / \beta}}
$$

where $\zeta(s)=\sum_{n=1}^{\infty} 1 / n^{s}$ is the Riemann zeta function. Fig. 1 compares the values of spatial reuse in these two cases for $T=$ $10 \mathrm{~dB}$ and different $\beta$. Note that in frequency-division multiple-access (FDMA) hexagonal networks with super-hexagonal frequency reuse, the spatial reuse is equal to $1 / \sqrt{3 K}$ where $K=3,4,7,12 \ldots$ is the possible cluster size (see [21, p. 285, eq. (3.20)]).

\section{Best Range Given Some MAP}

Assuming some given intensity $\lambda$ of transmitters, we will use the following notation and definition:

$$
\begin{gathered}
r_{\max }(\lambda)=\underset{r \geq 0}{\arg \max }\left\{r p_{r}(\lambda)\right\} \\
\rho(\lambda)=\max _{r \geq 0}\left\{r p_{r}(\lambda)\right\} .
\end{gathered}
$$

We call $r_{\max }(\lambda)$ the best range attempt for $\lambda$ and $\rho(\lambda)$ the best mean range. For exponential $S$, by Lemma $3.1,0<r_{\max }<\infty$, $0<\rho<\infty$. For a general $S$, we have the following technical result.

Lemma 4.5: If $\operatorname{Pr}(S>0)>0$ then $p_{r}(\lambda)$ is continuous in $r$. If moreover $\boldsymbol{E}\left[S^{2 / \beta}\right]<\infty$ then $\lim _{r \rightarrow \infty} r p_{r}(\lambda)=0$ for any $\lambda>0$.

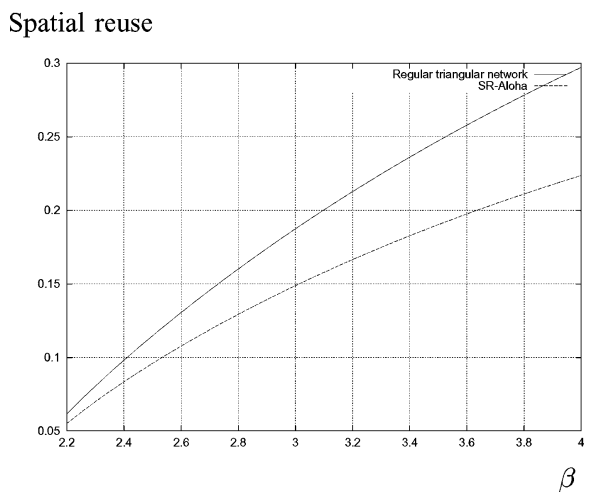

Fig. 1. Comparison of the spatial reuse factor for Poisson (lower curve) and perfect triangular network (upper curve) for $T=10 \mathrm{~dB}$ and different $\beta$. In hexagonal TDMA networks, with super-hexagonal frequency reuse and cluster size $K=3$ this parameter equal to $1 / 3=0$. (3) whereas for $K=7$ it is $1 / \sqrt{21}=0.218$ (regardless of $\beta$ ).

The proof is given in the Appendix . As a consequence of the preceding lemma, the function $r \mapsto \lambda p_{r}(\lambda)$ attains its maximum in $(0, \infty)$ and if we take $r_{\max }$ to be the value for which the spatial density of successful transmissions is maximal, then $0<$ $r_{\max }<\infty$.

By Lemmas 3.1, and 3.3 we have the following result.

Proposition 4.6: For a general $S$, the simplified attenuation function (3.1) and $W \equiv 0$

$$
\begin{aligned}
r_{\max }(\lambda) & =\frac{c_{3}}{T^{1 / \beta} \sqrt{\lambda}} \\
\rho(\lambda) & =\frac{c_{4}}{T^{1 / \beta} \sqrt{\lambda}}
\end{aligned}
$$

where the constants $c_{3}, c_{4}$ do not depend on $R, T, \mu$, provided $\lambda_{\max }$ is well defined. For exponential $S, c_{3}=1 / \sqrt{2 C}$ and $c_{4}=1 / \sqrt{2 e C}$.

Here again, trying to maximize the cumulated mean range of all transmissions initiated per unit of space w.r.t. $\lambda$, namely, trying to maximize $\lambda \rho(\lambda)$ in $\lambda$, leads to a degenerate answer since the maximum is for $\lambda=\infty$ which again gives $R=0$.

\section{Multihop Networks And Spatial Density of Progress}

We now return to the model of Section III with transmitters $\Phi^{1}$ and receivers $\Phi^{0}$ and focus on the multihop context.

\section{A. Progress}

Suppose a transmitter, say $X_{0}$, located at the origin $X_{0}=0$ has to send information in some given direction (say along the $x$ axis) to some destination located far from it (say at infinity - see Fig. 2). Since the destination is too far from the source to be able to receive the signal in one hop, the source tries to find a nontransmitting station in $\Phi^{0}$ such that the hop to this station maximizes the distance traversed toward the destination, among those which are able to receive the signal. This station will later forward the data to the destination or next intermediary station. In this model, the "effective" distance traversed in one hop, which we will call the progress, is equal to

$$
D=\max _{X_{j} \in \Phi^{0}}\left(\delta\left(0, X_{j}, \Phi^{1}\right)\left|X_{j}\right|\left(\cos \left(\theta\left(X_{j}\right)\right)\right)^{+}\right)
$$




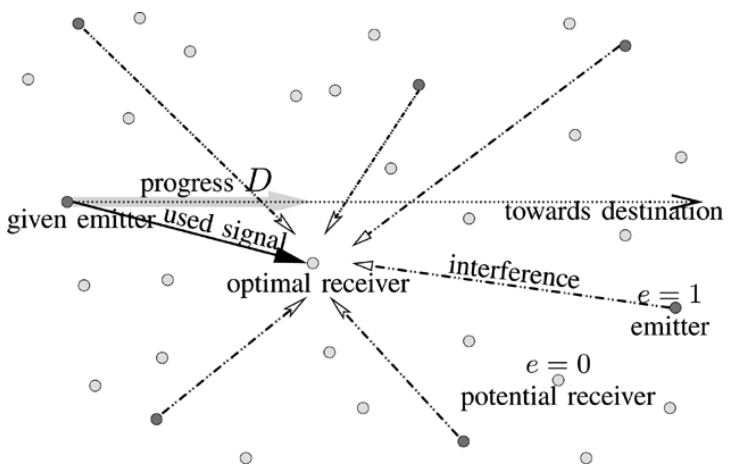

Fig. 2. Progress.

where $\theta(y)$ is the argument of the vector $y \in \mathbb{R}^{2}(-\pi<\theta(y) \leq \pi)$ and $\delta\left(x, y, \Phi^{1}\right)$ the indicator that (3.2) holds. We are interested in the expectation $d(\lambda, p)=\boldsymbol{E}[D]$ that only depends on $\lambda$ and on the MAP $p$, once given the parameters concerning emission and reception. Note that similarly to Proposition 4.1, we have the following formula for the (spatial) density of progress.

Proposition 5.1: The mean total distance traversed in one hop by all transmissions initialized in some unit area (density of progress) is equal to $\lambda p d(\lambda, p)$.

\section{B. MSR-Aloha and Optimal Progress}

Note that for a given $\lambda$, there is the following tradeoff in $p$ between the spatial density of communications and the range of each transmission. For a small $p$, there are few transmitters per unit area, although each of them can likely reach a very remote receiver as a consequence of the fact that $I_{\Phi^{1}}$ is small. On the other hand, a large $p$ means many transmitters per unit area that create interference and thus prevent each other from reaching a remote receiver. Another feature associated with large $p$ is the paucity of receivers, which makes the chances of a jump in the right direction smaller. In the following, we try to quantify this tradeoff and find $p$ that maximizes the density of progress. Since this optimization is adapted to the multihop context, the corresponding MAC protocol will be referred to as MSR-Aloha.

For mathematical convenience and also for reasons that will be discussed in Section VIII, we will not study $d(\lambda, p)$ directly but rather a surrogate (which is also a lower bound) of this quantity which we now introduce. Let

$$
\tilde{D}=\max _{X_{j} \in \Phi^{0}}\left(p_{\left|X_{j}\right|}(\lambda p)\left|X_{j}\right|\left(\cos \left(\theta\left(X_{j}\right)\right)\right)^{+}\right)
$$

and let $\tilde{d}(\lambda, p)=\boldsymbol{E}[\tilde{D}]$.

Proposition 5.2: For all $\lambda, p, d(\lambda, p) \geq \tilde{d}(\lambda, p)$.

Proof: Let $\boldsymbol{E}^{1}, \boldsymbol{E}^{0}$ denote expectation w.r.t. $\Phi^{1}$ and $\Phi^{0}$, respectively. Note that $\boldsymbol{E}[\tilde{D}]=\boldsymbol{E}^{1} \boldsymbol{E}^{0}[\tilde{D}]$ due to the independence between $\Phi^{1}$ and $\Phi^{0}$. The result now follows from Jensen's inequality, since the functional

$$
\varphi(f)=\boldsymbol{E}^{0}\left[\max _{X_{j} \in \Phi^{0}}\left(f\left(X_{j}\right)\left|X_{j}\right|\left(\cos \left(\theta\left(X_{j}\right)\right)\right)^{+}\right)\right]
$$

is convex on the space of real functions $f: \mathbb{R}^{2} \rightarrow \mathbb{R}$.
The aim of the remaining part of this section is to determine the value of the MAP $p$ that optimizes $\lambda p \tilde{d}(\lambda, p)$.

We will use the notation (cf Section IV-C)

$$
r_{\max }=r_{\max }(\lambda p)=\arg \max _{r \geq 0}\left\{r p_{r}(\lambda p)\right\}
$$

and

$$
\rho=\rho(\lambda p)=\max _{r \geq 0}\left\{r p_{r}(\lambda p)\right\} .
$$

For $z \in[0,1]$, let

$$
G(z)=\frac{2}{r_{\max }^{2}} \int_{\left\{r \geq 0: \rho z /\left(r p_{r}\right)<1\right\}} r \arccos \left(\frac{\rho z}{r p_{r}}\right) \mathrm{d} r .
$$

Remark 5.3: Note that if we assume the simplified attenuation model (3.1) and $W=0$, then Proposition 4.6 shows that $G(z)$ does not depend on the model parameters $\lambda, p, T, \mu$. Indeed, in this case

$$
G(z)=\frac{2}{c_{3}} \int_{\left\{r \geq 0: c_{4} z /\left(r \bar{p}_{r}\right)<1\right\}} r \arccos \left(\frac{c_{4} z}{r \bar{p}_{r}}\right) \mathrm{d} r .
$$

In particular, for exponential $S$, we have

$$
G(z)=2 \int_{\left\{t: e^{t} / \sqrt{2 e t} \leq 1 / z\right\}} \arccos \left(\frac{z e^{t}}{\sqrt{2 e t}}\right) \mathrm{d} t .
$$

We now study the distribution function of $\tilde{D}$.

Proposition 5.4: We have

$$
F_{\tilde{D}}(z)=\boldsymbol{P}(\tilde{D} \leq z)=e^{-\lambda(1-p)\left(r_{\max }(\lambda p)\right)^{2} G(z / \rho(\lambda p))} .
$$

Proof: Note in (5.2) that $\tilde{D}$ has the form of the so-called extremal shot noise $\max _{X_{i} \in \Phi^{0}} g\left(X_{i}\right)$ with the response function $g(x)=|x| p_{|x|}(\cos (\theta(x)))^{+}$. Its distribution function can be expressed by the Laplace transform of the (additive) shot noise

$$
\boldsymbol{P}\left(\max _{X_{i} \in \Phi^{0}} g\left(X_{i}\right) \leq z\right)=\boldsymbol{E}\left[\exp \left[\sum_{X_{i} \in \Phi^{0}} \ln \left(\mathbf{I}\left(g\left(X_{i}\right) \leq z\right)\right)\right]\right]
$$

and thus, for Poisson point process $\Phi^{0}$ with intensity $\lambda(1-p)$

$$
\boldsymbol{P}(\tilde{D} \leq z)=\exp \left[-\lambda(1-p) \int_{\mathbb{R}^{2}} \mathbb{1}(g(x)>z) \mathrm{d} x\right] .
$$

Passing to polar coordinates in the integral $\int_{\mathbb{R}^{2}} \ldots \mathrm{d} x$, we get

$$
\int_{\mathbb{R}^{2}} \mathbb{1}(g(x)>z) \mathrm{d} x=r_{\max }^{2} G(z / \rho)
$$

which completes the proof.

From Proposition 5.4, we immediately get the following.

Proposition 5.5: The expectation of $\tilde{D}$ is equal to

$\tilde{d}(\lambda, p)=\boldsymbol{E}[\tilde{D}]=\rho(\lambda p) \int_{0}^{1} 1-e^{-\lambda(1-p)\left(r_{\max }(\lambda p)\right)^{2} G(z)} \mathrm{d} z$. 
Corollary 5.6: For the model with the simplified attenuation function (3.1) and $W=0$, the expected modified progress is equal to

$$
\tilde{d}(\lambda, p)=\frac{c_{4}}{T^{1 / \beta} \sqrt{\lambda p}} H(p, T)
$$

and the spatial density of modified progress is

$$
\lambda p \tilde{d}(\lambda, p)=\frac{c_{4} \sqrt{\lambda p}}{T^{1 / \beta}} H(p, T)
$$

where

$$
H(p, T)=\int_{0}^{1} 1-\exp \left[\left(1-\frac{1}{p}\right) \frac{G(z)}{c_{3} T^{2 / \beta}}\right] \mathrm{d} z .
$$

Thus, the maximal density of progress is attained for the MAP $p^{*}=p^{*}(T)$ satisfying

$$
\sqrt{p^{*}} H\left(p^{*}, T\right)=\sup _{0 \leq p \leq 1} \sqrt{p} H(p, T) .
$$

For exponential $S$ this is equivalent to

$$
\int_{0}^{1}\left(1+\frac{G(z)}{p^{*} T^{2 / \beta} C}\right) \exp \left[\left(1-\frac{1}{p^{*}}\right) \frac{G(z)}{2 T^{2 / \beta} C}\right] \mathrm{d} z=1 .
$$

Note that $p^{*}$ does not depend on $\lambda$ and $\mu$.

\section{Numerical Examples and Discussion}

We now evaluate numerically the optimal MAP in the exponential case, and discuss the issue of the distance to the receiver that realizes the maximum in (5.2). This distance should not be large when one wants to implement the algorithm. We will show that at the optimal MAP $p^{*}$, the receiver that realizes the maximum in (5.2) is very likely in the vicinity of the transmitter. However, replacing the optimal receiver in (5.2) by the nearest one in some angle toward the destination gives an essentially suboptimal density of progress.

1) Numerical Approximations of $p^{*}$ : The successful numerical calculation of $\tilde{d}$ and of the solution $p$ of (5.8) maximizing the density of progress requires an efficient way of calculating the function $G$ given by (5.4). Below, we show some properties of $G$ that involve the so called Lambert $W$ functions $\mathrm{LW}^{0}$ and $\mathrm{LW}^{1}$. These functions can be seen as the inverses of the function $t e^{t}$ in the domains $(-1, \infty)$ and $(-\infty,-1)$, respectively; i.e., for $s \geq-1 / e, \mathrm{LW}^{0}(s)$ is the unique solution of $\mathrm{LW}^{0}(s) e^{\mathrm{LW}^{0}(s)}=s$ satisfying $\mathrm{LW}^{0}(s) \geq-1$, whereas for $0>s \geq-1 / e, \operatorname{LW}^{1}(s)$ is the unique solution of $\mathrm{LW}^{1}(s) e^{\mathrm{LW}^{1}(s)}=s$ satisfying $\operatorname{LW}^{1}(s) \leq$ -1 . Let

and

$$
L^{0}(s)=-\frac{1}{2} \mathrm{LW}^{0}\left(-s^{-2} e^{-1}\right)
$$

$$
L^{1}(s)=-\frac{1}{2} \operatorname{LW}^{1}\left(-s^{-2} e^{-1}\right)
$$

The following representation of $G$ is equivalent to that in (5.4):

$$
\begin{aligned}
G(z) & =2 \int_{L^{0}(1 / z)}^{L^{1}(1 / z)}\left(L^{1}(s)-L^{0}(s)\right) \mathrm{d} s \\
& =2 \int_{\arcsin (z)}^{\pi / 2}\left(L^{1}\left(\frac{\sin s}{z}\right)-L^{0}\left(\frac{\sin s}{z}\right)\right) \mathrm{d} s .
\end{aligned}
$$

Moreover, the following function:

$$
G_{\sim}(z)=\pi(1-z)-2 \ln (z) \arccos (z)
$$

approximates $G$ very well over the whole interval $0<z<1$. Fig. 3 shows the density of progress calculated by means of $G_{\sim}$

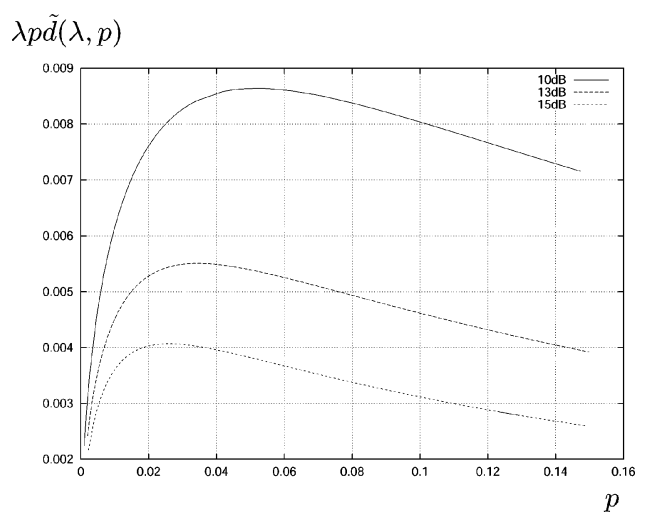

Fig. 3. Density of progress for the model with exponential $S$ and simplified attenuation function with $\beta=3, \lambda=1$, and $W=0$, with $T=\{10,13,15\}$ $\mathrm{dB}$ (curves from top to bottom). The optimal values (arg max, $\max$ ) are, respectively, $\{(0.052,0.0086),(0.034,0.0055),(0.026,0.0040)\}$.

for $\beta=3, \lambda=1$, and three values of the SINR threshold $T=\{10,13,15\} \mathrm{dB}$. On the plot, we can identify the MAP $p^{*}$ that maximizes the density of progress for a given $T$.

2) Location of the Optimal Receiver: First we will show that the optimal density of progress can be approached in the model with a reasonably restricted domain of reception. By this we mean that we exclude in the definition of $D$ and $\tilde{D}$ the receivers lying outside some disk with a given radius $R$. Note first that we have the following straightforward generalization of our previous results.

Proposition 5.7: Propositions 5.1, 5.2, 5.4, and 5.5 remain true if we take $\max _{X_{i} \in \Phi^{0},\left|X_{j}\right| \leq R}(\ldots)$ (with $\max \emptyset=0$ ) in definitions (5.1) and (5.2). In this case, the function $G$ has to be modified by taking the integral in (5.3) over the region $\left\{0 \leq r \leq R: \rho z /\left(r p_{r}\right)<1\right\}$. The case considered above will be referred to as the restricted range model in what follows.

We look for a reception radius $R$ such that for a given $p$, the density of progress in the restricted range model is close enough to that of the unrestricted range model. It is convenient to relate the reception radius $R$ with the intensity $\lambda$ of transmitters. As we will see later, it is even more convenient to take $R=K r_{\max }$ for some constant $K \geq 0$ (recall, that $r_{\max }=r_{\max }(\lambda p)$ is the distance at which the mean range $r p_{r}(\lambda p)$ is maximal). Denote by $G_{K}$ the function defined by (5.3) with the integral taken over $\left\{0 \leq r \leq K r_{\max }: \rho z /\left(r p_{r}\right)<1\right\}$.

We will continue with the simplified attenuation function (3.1) and $W=0$. In this case

$$
G(z)=\frac{2}{c_{3}} \int_{\left\{0 \leq<r \leq c_{3} K: c_{4} z /\left(r \bar{p}_{r}\right)<1\right\}} r \arccos \left(\frac{c_{4} z}{r \bar{p}_{r}}\right) \mathrm{d} r .
$$

We can now prove the following continuity result.

Proposition 5.8: For the simplified attenuation function and $W=0$

$$
0 \leq \tilde{d}-\tilde{d}_{K} \leq \rho z_{K}
$$

for some function $K \mapsto z_{K}$, such that $\lim _{K \rightarrow \infty} z_{K}=0$. For exponential $S$, we can take $z_{K}=K e^{1 / 2-K^{2} / 2}$ for $K \geq 1$.

Proof: Since $r \bar{p}_{r} \rightarrow 0$ when $r \rightarrow \infty$ (see the Appendix), for each $K \geq 0$, there exists $z_{K}$ such that $G_{K}(z)=G(z)$ for 
$z \geq z_{K}$. Moreover, $z_{K} \rightarrow 0$ when $K \rightarrow \infty$. Thus, (5.9) follows from Propositions 5.5 and 5.7.

Take for example, $p=0.035, T=13 \mathrm{~dB}$, and exponential $S$. In this case, the mean progress in the unrestricted model is approximately $\tilde{d}=0.0055 / 0.035=0.157$ (cf. Fig. 3), whereas the best mean range is attained for the range attempt $r_{\max }=$ 0.506 and is equal to $\rho=0.307$. In order to have a relative difference $\epsilon=0.01$ we find the minimal $K \geq 1$ such that

$$
K e^{1 / 2-K^{2} / 2} \leq 0.01 \cdot 0.157 / 0.307=0.00513
$$

which is $K=3.768$. This means that in the model with reception radius $R=K r_{\max }=3.768 \cdot 0.506=1.905$, the mean progress (and the density of progress) is within $1 \%$ of the optimal value obtained in the unrestricted model.

3) Comparison to Nearest Receiver in a Cone: It is easy to calculate the progress in the model when the transmitter chooses the nearest receiver in the cone of a given angle $\alpha$ toward the destination. Formally, let

$$
\tilde{D}_{\alpha}^{\text {near }}=p_{\left|X_{*}\right|}(\lambda p)\left|X_{*}\right| \cos \left(\theta\left(X_{*}\right)\right)
$$

where $X_{*}=X_{*}(\alpha)$ is such that

$$
\left|X_{*}\right|=\min \left\{\left|X_{j}\right|: X_{j} \in \Phi^{0},\left|\theta\left(X_{j}\right)\right| \leq \alpha / 2\right\} .
$$

Since the distribution function of $\left|X_{*}\right|$ for the Poisson process $\Phi^{0}$ with intensity $\lambda(1-p)$ is known to be

$$
\operatorname{Pr}\left(\left|X_{*}\right| \geq r\right)=\exp \left[-\lambda(1-p) \alpha r^{2} / 2\right]
$$

and since $\theta\left(X_{*}\right)$ is independent of $\left|X_{*}\right|$, uniformly distributed on $(-\alpha / 2, \alpha / 2)$, we easily get the following result on the mean progress in this scenario.

Proposition 5.9: For the simplified attenuation function, exponential $S$, and $W=0$ we have

$$
E\left[D_{\alpha}^{\text {near }}\right]=\frac{\Gamma(3 / 2)}{\sqrt{\lambda}} \frac{\sin (\alpha / 2)(1-p)}{\left((1-p) \alpha / 2+p T^{2 / \beta} C\right)^{3 / 2}} .
$$

Fig. 4 compares the density of progress $p \tilde{d}(1, p)$ in the "optimal receiver" case to the density of progress $p \boldsymbol{E}\left[D_{\alpha}^{\text {near }}\right]$ in the "nearest neighbor" case, for various values of $\alpha$ when $T=$ $10 \mathrm{~dB}$ and $S$ is exponential. The optimal choice of $\alpha$ is about $0.72 \pi$, for which the optimal MAP $p$ is about $p=0.056$ which gives $p \boldsymbol{E}\left[D_{\alpha}^{\text {near }}\right]=0.0080$, to be compared to $p^{*} \tilde{d}\left(1, p^{*}\right)=$ 0.0086 for $p^{*}=0.052$.

Finally, note in Fig. 5, that for $T$ in the range $0-10 \mathrm{~dB}$, the optimal value of the density of progress $p^{*} \tilde{d}\left(p^{*}, 1\right)$ is linear in $p^{*}$, which means that the mean progress $\tilde{d}\left(p^{*}, 1\right)$ does not depend greatly on $T$ in this range. We see on Fig. 6 that making $T$ very small increases the optimal MAP $p^{*}$ rather than the mean progress $\tilde{d}\left(p^{*}, 1\right)$.

\section{Continuity at $W=0$}

The results of Corollary 5.6 are obtained under the assumption $W=0$. We will show now that for a sufficiently small but positive $W>0$ the spatial density of progress $\lambda p d(\lambda, p)$ is also density of progress

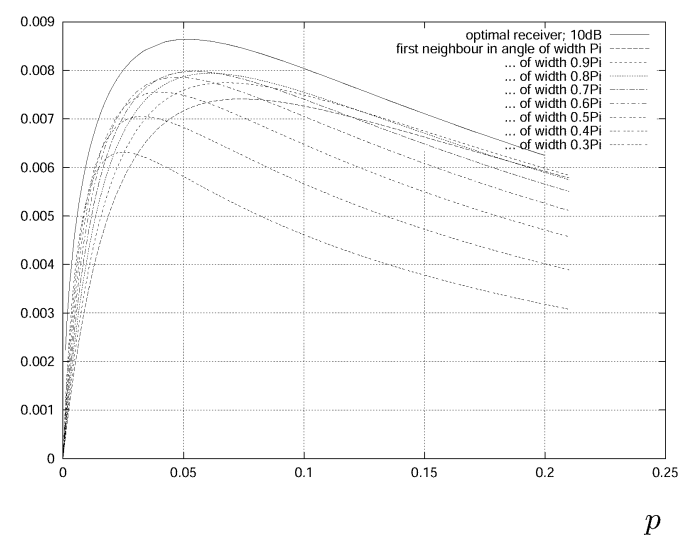

Fig. 4. Density of progress for the model with exponential $S$ and simplified attenuation function with $\beta=3, \lambda=1$, and $W=0$, with $T=10 \mathrm{~dB}$ for "optimal receiver" and "nearest neighbor" case.

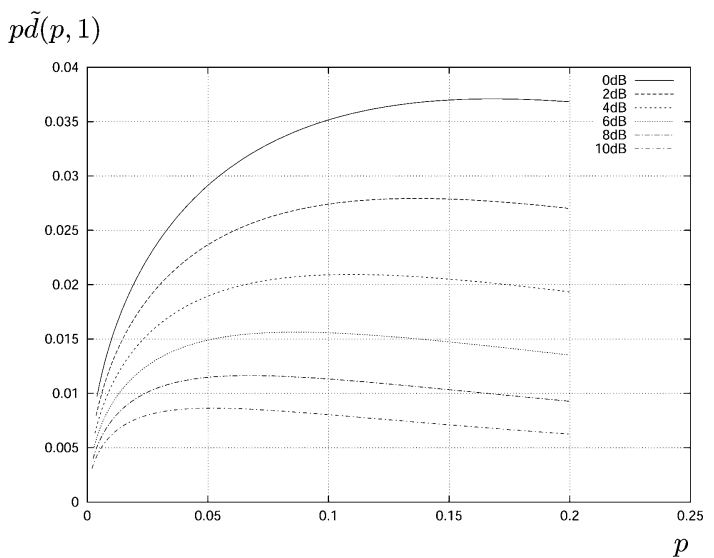

Fig. 5. Density of progress for the model with exponential $S$ and simplified attenuation function with $\beta=3$ and $W=0$ for moderate values of $T$.

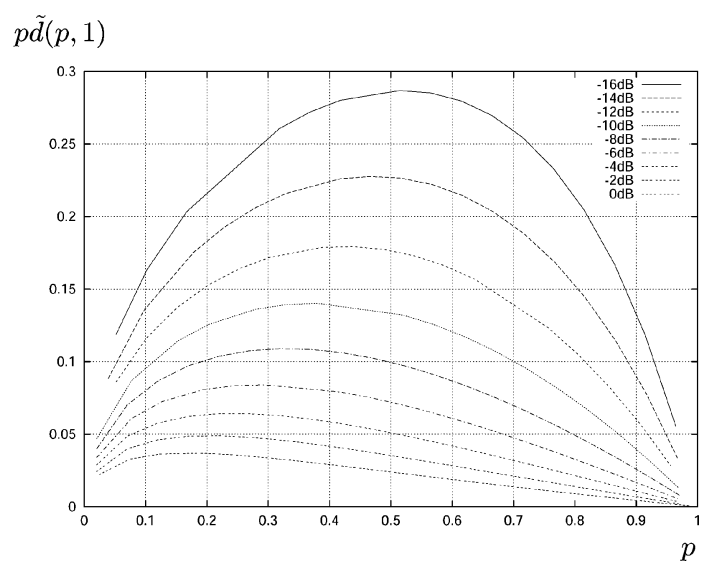

Fig. 6. Density of progress for the model with exponential $S$ and simplified attenuation function with $\beta=3$ and $W=0$ for small values of $T$.

of the order at least $\sqrt{\lambda}$ as $\lambda \rightarrow \infty$. Hence, the conclusions of the last sections are not due to a singular behavior at $W=0$.

Denote by $d(\lambda, p, w)$ the expected progress in the model with constant noise $W=w$. The following result is proven in the Appendix. 
Proposition 5.10: In a model with the simplified attenuation function and a fixed MAP $p$

$$
\lambda p d(\lambda, p, w)=\lambda p d(\lambda, p, 0)-\sqrt{\lambda} o\left(w \lambda^{-\alpha / 2}\right)
$$

when $w \lambda^{-\alpha / 2} \rightarrow 0$.

Corollary 5.11: For the simplified attenuation function, $W=w>0$, the optimal spatial density of progress is not less than

$$
\frac{c_{4} \sqrt{p^{*} \lambda}}{T^{1 / \beta}} H\left(p^{*}, T\right)\left(1+o\left(w \lambda^{-\alpha / 2}\right)\right)
$$

where $p^{*}$ is as in Corollary 5.6 and when $w \lambda^{-\alpha / 2} \rightarrow 0$.

Proof: We have

$$
\begin{aligned}
\sup _{p}(\lambda p d(\lambda, p, w)) & \geq \lambda p^{*} d\left(\lambda, p^{*}, w\right) \\
& =\lambda p^{*} d\left(\lambda, p^{*}, 0\right)-\sqrt{\lambda} o\left(w \lambda^{-\alpha / 2}\right) \\
& \geq \lambda p^{*} \tilde{d}\left(\lambda, p^{*}, 0\right)-\sqrt{\lambda} o\left(w \lambda^{-\alpha / 2}\right) \\
& =\frac{c_{4} \sqrt{p^{*} \lambda}}{T^{1 / \beta}} H\left(p^{*}, T\right)\left(1+o\left(w \lambda^{-\alpha / 2}\right)\right) .
\end{aligned}
$$

\section{CAPACITY AND STABiLITY}

In this section, we discuss capacity and stability issues for MSR-Aloha.

\section{A. Spatial Averages, Time Averages}

Up to now, we have analyzed spatial averages of the MSRAloha mechanism, such as, for instance, the spatial frequency with which a node experiences collisions. If nodes have no mobility, spatial averages do not coincide with time averages as some fixed node might experience a larger (resp., smaller) collision probability than the spatial average due to a particular configuration with many (resp., few) nodes in its neighborhood. We will return to the case without mobility in Section VII and now introduce the mobility assumptions used in what follows.

\section{B. Mobility Model}

The slotted mobility model that we use is close to the way point model: nodes are numbered in some way (e.g., using the distance to the origin at slot 0 ). Node $i$, which is located at $X_{i}^{n}$ in slot $n$ (the time slot is the slot of the Aloha scheme), has a random and independent motion vector $m_{i}^{n}$ during this slot, so that its position at slot $n+1$ is $X_{i}^{n+1}=X_{i}^{n}+m_{i}^{n}$. If the $\left\{m_{i}^{n}\right\}$ sequence is made of i.i.d. random vectors in $n$ and $i$, then $\left\{X_{i}^{n}\right\}$ is a Poisson point process at every time $n$ if it is at time 0 .

The law of $m_{i}^{n}$ is assumed to be nondegenerate (i.e., either the norm or the angle of this vector have a positive variance). This implies that the sequence of configurations seen by node $i$ over time (by configuration seen by mobile $i$ at time $n$, we understand the family of points $\left\{X_{j}^{n}-X_{i}^{n}\right\}_{j}$ ) is stationary and ergodic (see [22] for these definitions). In addition, for all Borel sets $A$ of the plane containing the origin and for all nonnegative functions $f$, the following almost sure limit holds for all $i$ :

$\lim _{n} \frac{1}{n} \sum_{k=1}^{n} f\left(\sum_{j} \mathbb{1}\left(X_{j}^{k}-X_{i}^{k} \in A\right)\right)=e^{-\lambda|A|} \sum_{p=0}^{\infty} \frac{(\lambda|A|)^{p}}{p !} f(p)$

where $|A|$ denotes the Lebesgue measure of $A$.
In other words, ergodic time averages (as given by the Cesaro limit in the left-hand side of the last equation) then coincide with spatial averages for a Poisson point process of intensity $\lambda$ (the right-hand side).

\section{Traffic Model}

In order to analyze the capacity of MSR-Aloha, we need to introduce a model for end-to-end communications, namely, for $\mathrm{o}-\mathrm{d}$ pairs in the plane and a traffic model for each such communication.

As we shall see, the following random segment model is appropriate for communications: each node is the origin of one communication. For each origin, one samples a random segment of the plane with uniform orientation and with random length of finite mean $L$; one centers one of its endpoints on the origin and selects as the destination the node of the Poisson point process that is the closest to the other segment endpoint. Note that the mean distance between a point of the Poisson point process and its nearest neighbor is proportional to $1 / \sqrt{\lambda}$, so that the ratio of the mean distance between origin and destination to the mean distance between nearest neighbors of this point process is proportional to $\sqrt{\lambda}$.

We will assume that each communication brings a mean value of $\tau$ fresh packets per slot. This defines a queuing network where each node has a queue of packets to be served/transmitted at the bit rate specified by the SINR threshold $T$. This queue is fed by packets which are either fresh packets originating from this node or packets arriving from another node and to be relayed.

Each node of this queuing network tries to transmit the packet head of the line with probability $p$ and either succeeds or keeps this packet head of line in case of collision (to be identified with a service completion with an instantaneous progress of $D=0$ ). The packet service rate of a node is hence $p$.

\section{Capacity of MSR-Aloha}

For MSR-Aloha, the end-to-end transport of each fresh packet requires a (spatial) average of $L / d(\lambda, p)$ one-hop transmissions ${ }^{2}$ when taking retransmissions due to collisions into account. Hence, the (spatial) average number of one-hop transmissions that are brought to the network per fresh packet and per unit of space is $\lambda L / d(\lambda, p)$. By homogeneity, the (spatial) average number of one-hop transmissions brought by the network to each station is hence $L / d(\lambda, p)$.

Since spatial averages are also time averages, $L / d(\lambda, p)$ is also the load brought by the network to any given node in the definitions of rate stability (see Appendix A).

We also know that when a node always has packets to transmit, the (time) average number of channel access is $p$. Hence, if the time intensity $\tau$ of fresh packets per node and slot is less than $p d(\lambda, p) / L$, then the network is rate-stable (see Section VI-A). Thus, under the above mobility assumptions, the rate-stability region of MSR-Aloha is

$$
\tau<C_{d}=\frac{p d(\lambda, p)}{L}
$$

\footnotetext{
${ }^{2}$ Note that this estimate assumes a perfect routing mechanism which might only make sense for cases of moderate mobility.
} 
at a given MAP $p$. From Proposition 5.2 and (5.6), we can approximate (and lower-bound) $C_{d}$ by

$$
C_{d} \geq \frac{c_{4} \sqrt{p}}{L T^{1 / \beta}} H(p, T) \frac{1}{\sqrt{\lambda}}=O\left(\frac{1}{\sqrt{\lambda}}\right)
$$

where $H(p, T)$ is given by (5.7). Choosing $p=p^{*}$ maximizes $\sqrt{p} H(p, T)$ and hence our surrogate (and lower bound) on $C_{d}$.

\section{E. Comparison With the Gupta and Kumar Random Model}

In [15], Gupta and Kumar consider a model with $n$ independent and uniformly distributed nodes in a disc (or sphere) of unit surface, which form an ad hoc network that is in charge of transporting bits between each node and its destination. For each origin node, the destination is randomly chosen in the disc.

Note that $n$ is also the intensity of the point process of nodes in this disc or sphere. Also note that the ratio of the mean distance between the origin and the destination to the mean distance between nearest neighbors of this point process is of the order of $\sqrt{n}$. This scale is similar to that of our model, so that the comparison of the Gupta and Kumar scheme and ours makes sense in spite of the differences between the models.

In [15], it is also assumed that each o-d pair is associated with a communication with a fresh packet rate $\tau$; so one can also associate a queuing network to the model in the same way as above. Gupta and Kumar analyze the mean load brought by a communication to a node and evaluate the minimum rate guaranteed to a given saturated node via and appropriate space-time scheduling. They obtain a rate-stability region of the form

$$
\tau<O\left(\frac{1}{\sqrt{n \log (n)}}\right)
$$

when $n \rightarrow \infty$. The last quantity is hence a constructive lower bound to the rate-capacity. It is also shown that the rate-capacity is bounded from above by $O(1 / \sqrt{n})$ when $n \rightarrow \infty$.

So, when identifying $n$ and $\lambda$, we conclude that MSR-Aloha achieves the optimal rate of $O(1 / \sqrt{\lambda})$ per source rather than $O(1 / \sqrt{\lambda \log (\lambda)})$ in Gupta and Kumar. A reason for this is that our protocol has no connectivity requirement. Indeed, it is for this requirement that Gupta and Kumar use a Voronoi tessellation with cells of mean diameter of order

$$
\rho(n)=O(\sqrt{\log (n) / n}))
$$

This scaling guarantees that, with probability approaching 1 as $n \rightarrow \infty$, each cell contains at least one node that can relay the traffic to some other node in one of the neighboring cells. As a consequence, in the scheme of Gupta and Kumar, the distance between a transmitter and its one-hop receiver is of the order of $\rho(n)$, that we know to be too large compared to the optimum $O(1 / \sqrt{n})$ found in the present paper. However, the following observations mitigate the fact that MSR-Aloha can sustain the optimal rate.

- This protocol requires mobility in order to achieve this rate.

- It intrinsically introduces random delays in relay nodes due to the randomness of the Aloha access scheme.

\section{F. Transport Capacity}

The spatial density of progress introduced above is closely related to Gupta and Kumar's [15] notion of transport capacity. The transport capacity of the network is defined as the number of bit-meters pumped every second by a unit area of the network. The constructive lower bound of [15] leads to a transport capacity of the order of $O\left(\frac{\sqrt{n}}{\sqrt{\log (n)}}\right)$ when $n \rightarrow \infty$.

The MSR-Aloha protocol also pumps a certain number of bit-meters every second. If the bit rate corresponding to the threshold $T$ is $b$, then the density of progress is $\lambda p d(\lambda, p)$ and MSR-Aloha progresses with a mean value of $C_{t}=b \lambda p d(\lambda, p)$ bit-meters per second and per unit area.

There are two important differences between the transport capacity and the density of progress. The first difference is the geometric nature of the latter, which measures the progress toward the destination rather then the magnitude of the jump in one hop. The second difference is the fact that the transport capacity is applicable to each part of the network, whereas the special density of progress is a spatial average that is only meaningful for a given part of the network when spatial averages coincide with time averages.

From Proposition 5.2 and (5.6), we can lower-bound the density of progress by

$$
C_{p}=b \frac{c_{4} \sqrt{p^{*}}}{T^{1 / \beta}} H\left(p^{*}, T\right) \sqrt{\lambda}=O(\sqrt{\lambda}) .
$$

\section{G. Dynamic Stability of MSR-Aloha}

By analogy with what we know of Aloha or Ethernet, a natural question is whether the rate-stability result obtained above implies the dynamic stability of the queuing network, namely, whether a time intensity $\tau$ of communications smaller than $C_{d}$ leads to a stable dynamic for this queuing network (it is well known that there exist queuing networks where rate stability is not enough to guarantee dynamic stability such as, for instance, rentrant lines or multiclass networks). By stable dynamics, we mean here the existence of a stationary regime for the queuing network given that the arrival processes of fresh packets are themselves stationary and ergodic. This question is open at this stage. We do not know of any results on the issue within the context of the lower bound scheme of [15] either.

\section{The Case Without Mobility}

We now show that in the no mobility case, MSR-Aloha provides a positive throughput and a positive progress to any node of the network and is still optimal in a sense defined below. The setting is as follows.

- $\Phi=\left\{X_{i}\right\}$ denotes the locations of nodes; we still assume an infinite number of nodes in the plane with locations that remain fixed for all time slots.

- The medium access sequence of station $i$ is an i.i.d. sequence $\left\{e_{n}^{i}\right\}$, independent of everything else, with value 1 with probability $p$ in slot $n$ if the station is allowed to transmit in this slot, and 0 otherwise.

- The potential powers of node $i$ is also an i.i.d. sequence $\left\{S_{n}^{i}\right\}$, independent of everything else, with some common 
distribution. We distinguish between two cases: that of a distribution with either unbounded or bounded support.

Each time $n$ when node $i$ is allowed to transmit, the interference for the signal transmitted by node $i$ at receiver $j$ is

$$
I_{\Phi}(n, i, j)=\sum_{k \neq i} S_{n}^{k} e_{n}^{k} l\left(\left|X_{j}-X_{k}\right|\right)
$$

Since the locations are fixed, this sequence is i.i.d.

Our only assumption in the unbounded support case is that the series

$$
J_{\Phi}(n, x)=\sum_{k} S_{n}^{k} l\left(\left|x-X_{k}\right|\right)
$$

is almost surely (a.s.) convergent for all $x$. A simple example where this assumption is satisfied is that where the locations $\left\{X_{i}\right\}$ are a realization of some homogeneous Poisson point process and the $\left\{S_{n}^{i}\right\}$ have a finite mean. It then follows from shot noise theory that the expectation (with respect to the Poisson law) of this series is finite so that the series itself is convergent indeed for a.s. all realizations of $\left\{X_{i}\right\}$.

In the bounded support case, we just assume that

$$
H_{\Phi}(x)=\sum_{k} l\left(\left|x-X_{k}\right|\right)
$$

is finite for all $x$. This is again satisfied for realizations of homogeneous Poisson point processes.

Let us now show that given $\left\{X_{i}\right\}$, the success of a transmission from $i$ to $j$ in slot $n$, namely, the event

$$
\left\{S_{n}^{i} l\left(\left|X_{j}-X_{i}\right|\right)>T I_{\Phi}(n, i, j)\right\}
$$

is of positive probability. This together with the fact that the sequence $\left(I_{\Phi}(n, i, j), S_{n}^{i}\right)$ is i.i.d. (in $\left.n\right)$ will in turns imply that the progress from node $i$ toward $j$ has a positive expectation and also that the transmission attempts from $i$ to $j$ succeed in infinitely many slots.

\section{A. Case 1: The Support of $S_{n}^{i}$ is Unbounded}

We have to prove that

$$
\boldsymbol{P}\left(S_{n}^{i} l\left(\left|X_{j}-X_{i}\right|\right)>T I_{\Phi}(n, i, j)\right)>0 .
$$

From the independence of $S_{n}^{i}$ and $I_{\Phi}(n, i, j)$, for all $x$

$$
\begin{aligned}
\boldsymbol{P}\left(S_{n}^{i} l\left(\left|X_{j}-X_{i}\right|\right)>T I_{\Phi}(n, i, j)\right) \\
\geq \boldsymbol{P}\left(S_{n}^{i} l\left(\left|X_{j}-X_{i}\right|\right)>x\right) \boldsymbol{P}\left(T I_{\Phi}(n, i, j) \leq x\right) .
\end{aligned}
$$

Since $I_{\Phi}(n, i, j)$ is a.s. finite, there exists an $x$ (that possibly depends on $i$ and $j$ ) such that $\boldsymbol{P}\left(T I_{\Phi}(n, i, j) \leq x\right)$; since $S_{n}^{i}$ has infinite support, then $\boldsymbol{P}\left(S_{n}^{i} l\left(\left|X_{j}-X_{i}\right|\right)>x\right)>0$ too, and this concludes the proof.

\section{B. Case 2: The Support of $S_{n}^{i}$ is Bounded}

Let us denote the maximal value of $S_{n}^{i}$ by $\sigma$ Let us show that for all positive real numbers $\eta$ the probability that the random variable $J_{\Phi}(n, x)$ is less than $\eta$ is positive: since the series $H_{\Phi}(x)$ is convergent and since

$$
J_{\Phi}(n, x) \leq \sigma H_{\Phi}(x)
$$

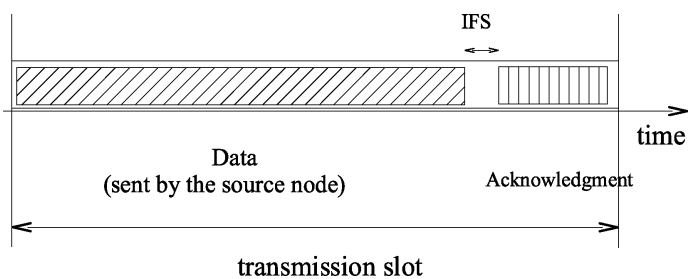

Fig. 7. Slot structure in MSR-Aloha.

then for all $\eta>0$, there exists a finite subset of the indices $F$ (that may depend on $i$ and $j$ but which does not depend on $n$ ) and such that the sum of all the terms of $J_{\Phi}(n, x)$ over the indices $k$ that do not belong to $F$ is less than $\eta$. Hence, the probability that $J_{\Phi}(n, x)$ is less than $\eta$ is larger than the probability that $e_{n}^{i}=0$ for all $i \in F$, which is positive since $F$ is finite. Using this and the fact that $S_{n}^{i}$ is independent of $I_{\Phi}(n, i, j)$, it is easy to check that again that

$$
\boldsymbol{P}\left(S_{n}^{i} l\left(\left|X_{j}-X_{i}\right|\right)>T I_{\Phi}(n, i, j)\right)>0 .
$$

Hence, MSR-Aloha provides a positive throughput to any node of any infinite network provided the interference created by all nodes in this network is finite at any point of the plane.

Let us return to the particular case where the locations of nodes are one fixed realization of some homogeneous Poisson point process with intensity $\lambda$, the powers are exponential of parameter $\mu$. In this case, the spatial average of the progress made over all network nodes in any given slot is still correctly evaluated by the stochastic geometry calculations of Section V-B. Hence, the spatial density of progress is still maximized by the choice of $p=p^{*}$. So in this case, MSR-Aloha is still optimal in this spatial average sense, although time averages of progress now fluctuate from node to node depending on the fixed environment seen by each node.

\section{IMPLEMENTATION ISSUES}

This section addresses the design issues of a MSR-Aloha MAC protocol based on the notion of progress. As described in the model, MSR-Aloha is a slotted protocol. The slots can be obtained via the timing information of a positioning system such as the global positioning system (GPS) or local atomic clocks (cesium-beam, rubidium clocks or hydrogen maser clocks) can provide nodes with such a synchronization. MSR-Aloha being a random-access MAC protocol, we also have to cope with collisions. Of course, MAC collisions can be handled above the MAC layer but it can be easily shown that this leads to inefficient communication systems. This is why a good implementation of MSR-Aloha should use MAC acknowledgments for point-to-point packets as is done in MAC protocols used for WLAN's standards [2], [3]. We have assumed that MSR-Aloha is slotted. The slot can be divided into two parts: a data part (the main part) used by the transmitter to send the packet and an acknowledgment part used by the receiver to indicate that it has received the packet correctly (see Fig. 7). There is an issue concerning the correct reception of the acknowledgment since the global geometry of the transmissions of acknowledgments 
is different from that of the transmission of the data packets. This issue can be solved by using code-division multiple-access (CDMA) codes to send the acknowledgments. Each data packet mentions the CDMA code with which the recipients have to reply. As we will see later, all the receivers of a given packet will use this one code, and if the number of available CDMA codes is large enough, a random selection amongst available CDMA codes will make collision in codes of neighboring packet transmissions very unlikely. Since gains of more than $10 \mathrm{~dB}$ are very easy to build, the correct reception of acknowledgments is very likely. If a packet is not correctly acknowledged, MSR-Aloha will just have to send the packet again still using $p$ as transmission probability.

Actually, the MAC transmission policy of MSR-Aloha is extremely simple; whenever an MSR-Aloha node has a packet to send or to retransmit, it must send it using $p$ as transmission probability on each slot. The reception of an acknowledgment packet is used to qualify the correct transmission of a packet. Computation of $p$ can be done $a$ priori since it is only necessary to know the capture threshold $T$. Thus, no special channel monitoring is needed.

Since MSR-Aloha is optimized for a multihop network, MSR-Aloha must be closely related to a routing protocol. It is beyond the scope of this paper to describe routing algorithms or to fully study how routing algorithms could work with MSR-Aloha. Most existing routing protocols do not use the geographical locations of nodes to compute routes, but research has shown that geographical location information can improve routing performance in mobile multihop networks [23], [24]. In the following, we give a few hints concerning the use of MSR-Aloha with geographical position information-assisted routing protocols.

We can imagine two techniques for MSR-Aloha: the next hop toward the final destination is directly computed or it is the result of a real transmission.

\section{A. Direct Computation of the Next Hop}

For this solution, it will be assumed that each network node knows the locations of all network nodes including itself. Thus, the transmitter knows its location (say 0), the direction of the final destination, and the locations $X_{i}$ of the transmitter's neighbors expressed in the referential centered in the transmitter in 0 and such that the $x$ axis points to the destination. It can hence evaluate the functions $p_{\left|X_{i}\right|} v\left(X_{i}\right)$ for all $i$, where $v(x)=|x|(\cos (\theta(x)))^{+}$and determine which is the best neighbor to be the next hop toward the final destination.

Notice that this algorithm can also be implemented by the receivers. As a matter of fact, the functions $p_{\left|X_{i}\right|} v\left(X_{i}\right)$ can also be (pre)computed by the receivers. The receiver that realizes the maximum of this function can elect itself as the next hop to the final destination. In either case (the transmitter selects the next hop or the next hop selects itself), an acknowledgment must be sent by the receiver to the transmitter. Notice that

- such a direct computation of the next hop realizes the mean optimal progress (5.2);

- the function $r \mapsto p_{r}(\lambda p)$ must be known;

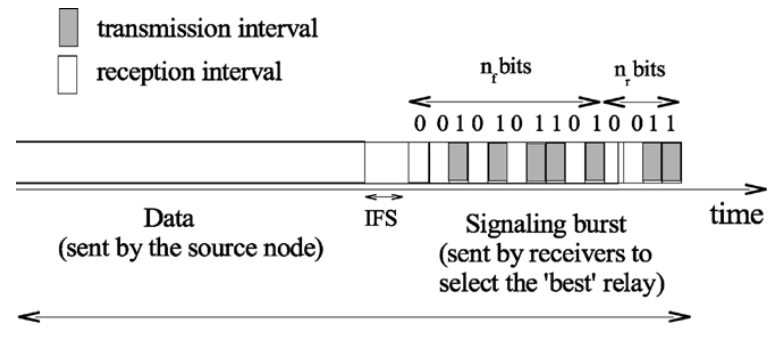

transmission slot

Fig. 8. Active signaling technique. When a burst is detected in a reception interval, the node quits the selection process. Thus, the selected receiver ("best relay") will be the receiver having used the greatest binary sequence for its signaling burst.

- the actual optimization requires not only knowledge of the location of the nodes, but also their actual MAC states (either receiver or transmitter), which is an unrealistic assumption. Notice, however, that the lack of information on the MAC state of other stations may only be problematic when the station that is elected to relay a packet happens to be a transmitter in the considered slot. Given that $p$ is rather small, this is a relatively rare event that should perhaps simply be interpreted as a collision.

For this solution, we have assumed that each node knows its location and the locations of all the other nodes. Although actually only the locations of the neighbor nodes and the destination node need to be known, we cannot claim that this scheme is completely independent of the network density $\lambda$. The following solution will have this property.

\section{B. Next Hop Selected in a Real Transmission}

We are looking for a mechanism which can at the same time acknowledge the reception of the current transmission and select among the potential receivers the one which offers the greatest progress toward the destination. Such a mechanism can be implemented using an active signaling scheme in receivers similar to the scheme used in the Hiperlan type 1 [3] access technique called elimination yield nonpre-emptive multiple access (EY-NPMA). Note that EY-NPMA can be precisely analyzed in a single-hop context, see [25].

The transmission slot is divided into a main part used by the transmitter to send the data and the remaining part of fixed length at the end of the slot which is used by the potential receivers. In this remaining part of the slot, the potential receivers (i.e., these who have successfully received the packet, and one of whom will forward it as the best relayer) send a burst of active signaling used for the selection of the best receiver. This burst is composed of a sequence of intervals of the same length in which a given receiver can either transmit or listen (see Fig. 8). During this active signaling phase, each receiver applies the following rule: if it senses a signal during any of its listening intervals, it quits the selection process (namely, it stops transmitting throughout the remaining part of the active signaling phase). The reason for this stems from the construction of signaling bursts (described below): the sensing of a transmission during a listening interval implies that a better relay has also correctly received the data information sent in the first part of the slot. 
1) Signaling Burst: Let us now describe the way signaling bursts are built. Each such burst is best represented by a binary sequence where 1 denotes a transmission interval and 0 denotes a listening one. This binary sequence is computed by each reception node as follows: the first $n_{f}$ bits are computed by the receiver as a function of the progress the node offers as a relay to the packet. Since we assume that the data packet includes the address of the source and the address of the final destination, a node can easily compute this progress it offers as relay to a received packet. For instance, we can assume that the first $n_{f}=$ 16 bits gives the progress, for instance in meters, offered by the relay coded in base 2 . With such figures, progress ranging from $1 \mathrm{~m}$ to $65 \mathrm{~km}$ can be declared; this covers most of practical network configurations. It is easy to check that if the progress offered by a receiver 1 is larger than that of receiver 2 , then there exists an interval in which receiver 2 listens and receiver 1 transmits, which is exactly the announced property. We add $n_{r}$ bits selected at random to discriminate between nodes offering the same progress. We will also assume that the sequence encompasses a last bit set to 1 . This bit forces the receiver which remains active after the selection process to provide evidence of its activity. Thus, if the transmitter (the node that sent the data packet in the first part of the slot) cannot sense a signal in the last interval of the signaling burst, it infers that its packet has not been received or that the selection process between potential relays has failed and the data packet must be retransmitted according to the Aloha rule. To cope with interference between several selection processes taking place in different locations of the plane during the same signaling burst, it is recommended to use CDMA codes; the code to be used by all receivers of a given packet to acknowledge this packet and select the best relay that can be provided in the packet.

There remain two issues concerning the autoselectionacknowledgment process.

2) Length of the Signaling Burst: First, the binary sequence of the active signaling used for the selection of the optimal receiver should be long enough to be able to discriminate between all the potential receivers. A brisk analysis shows that the expected number of successful receivers of a given packet is $O(1)$ when $\lambda \rightarrow \infty$ and thus it is possible to fix a length of this binary sequence that will be sufficient for all $\lambda$. Indeed, for the simplified attenuation function and $W \equiv 0$, by Lemma 3.3, this expected number is equal to

$$
\begin{aligned}
\boldsymbol{E}\left[\sum_{X_{i} \in \Phi^{0}} \delta\left(0, X_{i}, \Phi^{1}\right)\right] & =\lambda\left(1-p^{*}\right) \int p_{|x|}\left(\lambda p^{*}\right) \mathrm{d} x \\
& =\frac{1-p^{*}}{p^{*} T^{2 / \beta}} \int \bar{p}_{|x|} \mathrm{d} x .
\end{aligned}
$$

3) Interference in the Signaling Burst: The second issue concerns the interference created in the active signaling phase. In Fig. 9, we have shown simultaneous transmissions with their related receiving nodes. The aim of the autoselection-acknowledgment is to select the best "relay" toward a given final destination. The signaling technique used to perform this selection generates interference.

It is beyond the scope of this paper to give a detailed stochastic-geometry analysis of this problem. Instead, we will briefly explain why it is possible to fix a CDMA code length

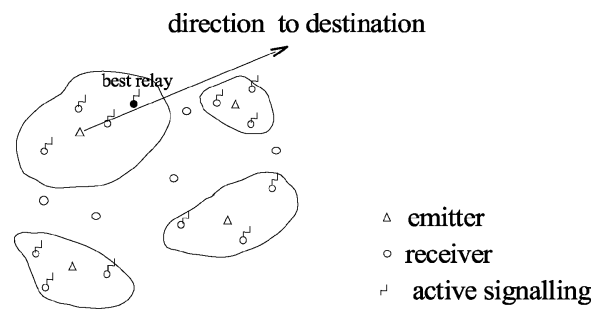

Fig. 9. Simultaneous transmissions with their receiving areas. The active signaling scheme used in a receiving area to select the "best" relay will generate interference in other receiving areas.

that provides enough orthogonality to cope with interference in this phase, for all $\lambda>0$. Note that there are two possible misbehaviors due to the interference in this phase.

a) One is when a potential receiver, in one of its listening intervals, does not correctly receive the signal coming from one of his competitors. In such a case, it may infer that there is energy from another transmission attempt and that, actually, there is a signaling burst sent by a better relay for this very transmission.

b) Another is when a potential receiver (or even the transmitter when it looks at the last interval of the signaling burst) takes the interference resulting from the signaling burst of an other autoselection process as a signaling burst for its own signaling process.

First Problem. Correct receptions must be validated on an SINR basis. The following approximation/bound of the probability of the correct reception can be considered:

$$
p_{R^{\text {ack }}}^{\text {ack }}=\operatorname{Pr}\left(S^{\text {ack }} \geq \gamma T^{\text {ack }} I^{\text {ack }}\left(A R^{\text {ack }}\right)^{\beta}\right)
$$

where $S^{\text {ack }}$ is the power used in active signaling, $T^{\text {ack }}$ is the SINR threshold, $R^{\text {ack }}$ is the distance over which the right signal is attenuated, $I^{\text {ack }}$ is the interference in this phase, and $\gamma$ is the orthogonality factor due to usage of the CDMA codes of a given length. Note that, due to our previous considerations, $R^{\text {ack }}=O(1 / \sqrt{\lambda})$ and $I^{\text {ack }} \approx \sum_{X_{i} \in \Phi^{1}} N_{i} S_{i}^{\text {ack }}\left(A\left|X_{i}\right|\right)^{-\beta}$, where $N_{i}=O(1)(\lambda \rightarrow \infty)$ is the number of potential receivers of the packet transmitted by the transmitter $X_{i}$. Thus, for the simplified attenuation function and $W \equiv 0$, by Lemma 3.3

$$
p_{R^{\text {ack }}}^{\text {ack }}=\bar{p}_{O(1 / \sqrt{\lambda})}^{\text {ack }} \sqrt{\lambda p^{*}}\left(\gamma T^{\text {ack }}\right)^{1 / \beta}
$$

where $\bar{p}_{R}^{\text {ack }}$ denotes the probability of success for the model with $\gamma=T^{\text {ack }}=\lambda=1$. This shows that $p_{R^{\text {ack }}}^{\text {ack }}=O(1)$ when $\lambda \rightarrow \infty$ and moreover, $p_{R^{\text {ack }}}^{\text {ack }} \rightarrow 1$ when $\gamma \rightarrow 0$.

Second Problem. This problem cannot be validated on a SINR basis. We have to fix an absolute threshold for the power of the signal received in the autoselection process, based on which the user will be able to distinguish between the burst of its own signaling process and a burst of a different autoselection. In order to make the process decentralized and autoadapting to the density $\lambda$, we let each receiver $X_{i}$ fix this threshold as some fraction $\tau$ of the power $S\left(A\left|X_{i}\right|\right)^{-\beta}$ it received from the transmitter in the data part of the transmission slot. The fraction $\tau$ should be set to a value such that the probability $P_{1}$ of the detection of the signaling process associated with its own emission is great, while the probability $P_{2}$ of the detection 
of a burst from a different autoselection is small. These two probabilities can be approximated/bounded as follows:

$$
\begin{aligned}
& P_{1}=\operatorname{Pr}\left(S^{\text {ack }}\left(A R^{\text {ack }}\right)^{-\beta} \geq \tau S\left(A\left|X_{*}\right|\right)^{-\beta}\right) \\
& P_{2}=\operatorname{Pr}\left(I^{\text {ack }} \geq \tau S\left(A\left|X_{\dagger}\right|\right)^{-\beta}\right)
\end{aligned}
$$

where we take $X_{*}$ to be the nearest user to the transmitter (which determines the largest threshold) and $X_{\dagger}$ to be the most remote user in the cluster of receivers participating in the autoselection (which determines the smallest threshold). Assuming $S^{\text {ack }} \approx S$ and knowing that $R^{\text {ack }}=O(1 / \sqrt{\lambda})$ we have

$$
\begin{aligned}
P_{1} & =\operatorname{Pr}\left(\left|X_{*}\right| \geq \tau^{1 / \beta} R^{\text {ack }}\right) \\
& =e^{-\lambda\left(1-p^{*}\right) \tau^{2 / \beta}\left(R^{\text {ack }}\right)^{2}} \\
& =e^{-\left(1-p^{*}\right) \tau^{2 / \beta} O(1) \quad(\lambda \rightarrow \infty)}
\end{aligned}
$$

and we can take $\tau>0$ small enough to make $P_{1}$ close to 1 . For the second probability, assuming $S^{\text {ack }} \approx S$ and knowing that $\left|X_{\dagger}\right|=O(1 / \sqrt{\lambda})$, we have

$$
\begin{aligned}
P_{2} & =1-\bar{p}_{\left|X_{\dagger}\right| \sqrt{\lambda p^{*}}(\gamma / \tau)^{1 / \beta}}^{\text {ack }}, \quad(\lambda \rightarrow \infty) \\
& =1-\bar{p}_{\sqrt{p^{*}}(\gamma / \tau)^{1 / \beta} O(1)}^{\text {ack }} \quad
\end{aligned}
$$

and we can take $\gamma>0$ large enough to make $P_{2}$ close to 0 .

4) Summary: The receiver selection version of the MSRAloha protocol has the following interesting properties:

- for any given MAP $p$, it realizes a mean progress $d(\lambda, p)$ larger than that of the direct computation method $(\tilde{d}(\lambda, p)$; see Result 5.2);

- its throughput scales in at least $O(\sqrt{\lambda})$ (this follows from the last inequality and from the results of Section V-B);

- the protocol does not require that $p_{r}(\lambda p)$ or even $\lambda$ be known (incidentally, the authors do not know of any other protocol that has this property);

- there are no extra connectivity requirements (which explains why its throughput is in $O(\sqrt{\lambda})$ and not in $O\left(\frac{\sqrt{\lambda}}{\sqrt{\log (\lambda)}}\right)$ ) and hence no need of neighborhood management;

- it is fully decentralized and it scales to arbitrarily large configurations (as shown by the mathematical analysis that considers an infinite number of nodes scattered through the whole plane with any given density).

\section{CONCLUSION}

We have introduced a spatial reuse Aloha multiple-access protocol adapted to large random homogeneous mobile networks using multihop transport mechanisms. Thanks to a direct representation of the interference process and of the progress made by each transmission, we have shown how the transport capacity of the network could be maximized by selecting the probability of channel access appropriately. We have shown that the transport capacity of such a network is proportional to the square root of the density of nodes under the assumption that there is some nondegenerate node mobility. Among the most interesting properties of this protocol, we would primarily point to the fact that the optimal value of its parameter does not depend on node intensity and the fact that the protocol can be implemented in a fully distributed way.

\section{APPENDIX}

\section{A. Rate Stability}

Consider a queuing network with a single class of customers. Each customer entering the network has a route that consists of some random sequence of nodes of the queuing network, and a sequence of service requirements along this route. Let $\rho_{i}$ denote the (time-ergodic) mean service load brought by a customer to node $i$. By time-ergodic mean, we understand Cesaro mean values over time which are assumed to exist and to coincide with the mathematical expectation. Let $\alpha_{i}$ denote the saturation rate of node $i$ which is defined as the (time-ergodic) rate at which this node serves packets when it has an infinite backlog.

This network is said to be rate-stable if for all $i, \rho_{i}<\alpha_{i}$

\section{B. Tentative Comparison of SR-Aloha and CSMA}

The aim of this section is a tentative comparison between SR-Aloha and a generic CSMA protocol. Throughout the section, we assume a random Poisson network, the simplified attenuation function (3.1), and $W=0$. We suppose that the radius of the carrier sense range $R_{c s}$ is set at

$$
R_{c s}=R T^{1 / \beta} \frac{2(6 \zeta(\beta-1))^{1 / \beta}}{\sqrt{3}}
$$

where $R$ denotes the targeted transmission range. According to [20], there will be no collision for a receiver in a radius of range $R$ if the transmitters in the network are on a triangular regular network, i.e., network nodes are at the vertices of equilateral triangles generated by an initial equilateral triangle infinitely replicated by symmetry. Since in a triangular regular network the density of nodes being at least at $R_{c s}$ away is maximum, we conjecture that whatever the pattern of simultaneous emitters respecting the CSMA rule with $R_{c s}$, a transmission to a receiver within radius $R$ will always be collision free.

In order to compare SR-Aloha to the CSMA protocol, we have to compute the intensity of an extracted point process satisfying the CSMA exclusion rule. Of course, the intensity of this process will depend on the selection algorithm. An intuitive algorithm consists in picking nodes randomly and adding them to the CSMA transmission set if they are not in the carrier sense range of an already selected node. This algorithm is close to the effective behavior of a simple CSMA system. However, this model does not seem to be easily tractable mathematically. Another selection algorithm is that based in the Matern hard-core process [26], [27]. This process is a thinning of the initial Poisson point process in which points are selected according to random marks. A point of the process is selected if its mark is larger than all marks in a radius of range $R_{c s}$. It is easy to check that the selected points follow the CSMA rule. The spatial intensity $\lambda_{R_{c s}}$ of the Matern hard-core process can be obtained as a function of the spatial intensity of the initial Poisson point process by the formula

$$
\lambda_{R_{c s}}=\frac{1-e^{-\pi \lambda R_{c s}^{2}}}{\pi R_{c s}^{2}}
$$

(see [27]).

Simulations show that the intensity of this process is smaller than the intensity obtained through the random pick algorithm 


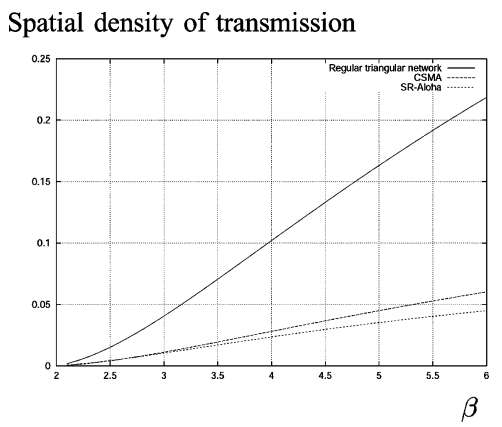

Spatial density of transmission

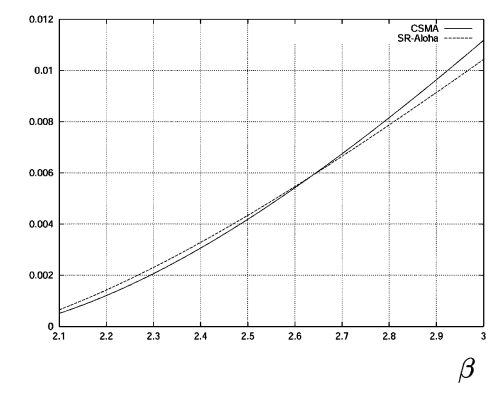

Fig. 10. Top: spatial intensity of successful transmissions for CSMA (Matern selection model) and for SR-Aloha scheme as a function of $\beta, T=$ $10 \mathrm{~dB}$. The top curve gives the throughput of a regular triangular network. Bottom: Zoom of the comparison CSMA-SR-Aloha for $\beta$ between 2 and 3 .

alluded to above, while giving results of the same order of magnitude. We can notice that the Matern hard-core process is a natural model for the access scheme of HiPERLAN type 1 . The MAC of HiPERLAN type 1 actually uses an advanced version of CSMA. A signaling burst is sent before the packet; the (random) length of this elimination burst will be the mark which allows the Matern process to be derived.

Since we know $R_{c s}$, it is easy to compute the transmission density for a CSMA scheme and to compare it with the spatial density of successful transmission of our SR-Aloha scheme given by Proposition 4.3.

This comparison is given in Fig. 10. Fig. 10 (top) compares the spatial intensity of CSMA (selection of active nodes as in a Matern hard core process) and the spatial density of successful transmissions of SR-Aloha scheme as a function of $\beta$, for $T=$ $10 \mathrm{~dB}$. The curve at the top gives the spatial intensity of CSMA in a regular triangular network. On the bottom we have a zoom for $\beta$ between 2 and 3 . We see that, near 2, the optimized Aloha scheme actually outperforms the CSMA scheme.

Fig. 10 shows that under these assumptions, the performance of SR-Aloha is very close to that of the CSMA scheme. This observation is consistent with [6], where a similar result reports that Aloha and CSMA have close performance. However the study in [6] uses a simplified transmission model (interference is only considered to propagate two hops away) and the carrier sense range and transmission range are supposed to be the same. In [28] a convenient tuning of the carrier sense range is shown to be important for the global performance of the network.

As a result of this tentative comparison we can conclude that SR-Aloha and a generic CSMA algorithm will have comparable performances. Further studies will be necessary to compare them more precisely.

\section{Proofs}

1) Proof of Lemmas 4.2 and 4.5: We give sufficient conditions for

$$
\begin{aligned}
p_{r}(\lambda) & =\operatorname{Pr}\left(S \geq T\left(W+I_{\Phi}\right)(A r)^{\beta}\right) \\
& =\operatorname{Pr}\left(r \leq \frac{S^{1 / \beta}}{A\left(T\left(W+I_{\Phi}\right)\right)^{1 / \beta}}\right)
\end{aligned}
$$

to be a continuous function of $r$ and $\lambda$ and for

$$
\lim _{\lambda \rightarrow \infty} \lambda p_{r}(\lambda)=\lim _{r \rightarrow \infty} r p_{r}(\lambda)=0 .
$$

Proposition A.1: If $\operatorname{Pr}(S>0)>0$ then the Poisson shot noise $I_{\Phi}$ is absolutely continuous w.r.t. the Lebesgue measure (has a density), consequently the same is true for $\frac{S^{1 / \beta}}{A\left(T\left(W+I_{\Phi}\right)\right)^{1 / \beta}}$ and hence, $p_{r}(\lambda)$ is continuous in $r$ and, by Lemma 3.3, in $\lambda$.

For the proof see [29, Proposition A.2].

Proposition A.2: Suppose $\operatorname{Pr}(S>0)>0$. If $\boldsymbol{E}\left[S^{2 / \beta}\right]<\infty$ then

$$
\lim _{r \rightarrow \infty} r p_{r}(\lambda)=0 .
$$

If $E\left[S^{3 / \beta}\right]<\infty$ then

$$
\lim _{\lambda \rightarrow \infty} \lambda p_{r}(\lambda)=0 .
$$

Proof: Note by (A1) that for

$$
\lim _{r \rightarrow \infty} r p_{r}(\lambda)=0
$$

it suffices to have

$$
E\left[\frac{S^{2 / \beta}}{A\left(T\left(W+I_{\Phi}\right)\right)^{2 / \beta}}\right]<\infty
$$

whereas for

$$
\lim _{r \rightarrow \infty} \lambda p_{r}(\lambda)=\lim _{r \rightarrow \infty} \lambda p_{r \sqrt{\lambda}}(1)=0
$$

it suffices to have

$$
E\left[\frac{S^{3 / \beta}}{A\left(T\left(W+I_{\Phi}\right)\right)^{3 / \beta}}\right]<\infty .
$$

The result follows from independence of $S, W, I_{\Phi}$ and from the fact that if $\operatorname{Pr}(S>0)>0$ then $\boldsymbol{E}\left[I_{\Phi}^{-\gamma}\right]<\infty$ for any $\gamma>0$. Indeed, take $\epsilon>$ such that $\operatorname{Pr}(S>\epsilon)>0$ and observe that $I_{\Phi} \geq I^{\prime}$ where $I^{\prime}=\epsilon\left(A\left|X_{*}\right|\right)^{-\beta}$, where $X_{*}$ is the point $X_{i}$ of $\Phi$ which is the closest to 0 and such that $S_{i}>\epsilon$. The distribution function of $\left|X_{*}\right|$ is

$$
\operatorname{Pr}\left(\left|X_{*}\right| \geq r\right)=e^{-\lambda \operatorname{Pr}(S>\epsilon) \pi r^{2}}
$$

and it is easy to see that

$$
\boldsymbol{E}\left[I^{\prime-\gamma}\right]=\epsilon^{-\gamma} A^{\gamma \beta} \boldsymbol{E}\left[\left|X_{*}\right|^{\gamma \beta}\right]<\infty
$$

for any $\gamma>0, \beta>0$.

2) Proof of Proposition 5.10: We have

$\lambda p d(\lambda, p, 0)-\lambda p d(\lambda, p, w)$

$$
\leq \boldsymbol{E}\left[\sum_{x_{i} \in \Phi^{1} \cap A} D_{x_{i}}^{0} \mathbb{I}\left(D_{x_{i}}^{0} \neq D_{x_{i}}^{w}\right)\right]
$$

where $A$ is a disc of area 1 and $D_{x_{i}}^{0}, D_{x_{i}}^{w}$ are the progresses realized by the node $x_{i}$ in, respectively, absence and presence 
of the noise $W=w$. By the Campbell formula, the expression in (A2) can be written as

$$
\lambda p \boldsymbol{E}\left[D^{0} \mathbf{I}\left(D^{0} \neq D^{w}\right)\right]
$$

where $D^{0}, D^{w}$ are the respective progresses for a typical node of $\Phi^{1}$ located at the origin. By the scaling property of the model with a simplified attenuation function (see the proof of Lemma 3.3) we can prove that

$$
\boldsymbol{E}\left[D^{0} \mathbf{I}\left(D^{0} \neq D^{w}\right)\right]=\frac{1}{\sqrt{\lambda}} o\left(w \lambda^{-\alpha / 2}\right)
$$

as $w \lambda^{-\alpha / 2} \rightarrow 0$

\section{REFERENCES}

[1] N. Abramson, "The Aloha system-Another alternative for computer communication," Proc. AFIPS, pp. 295-298, 1970.

[2] IEEE 802.11 Standard. Wireless LAN Medium Access Control (MAC) and Physical Layer (PHY) Specifications, 1997.

[3] ETSI HIPERLAN Functional Specifications, ETS 300-654, 1996.

[4] R. Rivest, "Network Control by Bayesian Broadcast," MIT, Lab. Comput. Sci., Cambridge, MA, Rep. MIT/LCS/TM-285.

[5] B. Hajek and T. Van Loo, "Decentralized dynamic control of a multiaccess broadcast channel," IEEE Trans. Autom. Control, vol. AC-27, no. 3, pp. 559-569, Jun. 1982.

[6] R. Nelson and L. Kleinrock, "Maximum probability of successful transmission in a random planar packet radio network," in Proc. IEEE INFOCOM, San Diego, CA, Apr. 1983, pp. 365-370.

[7] S. Ghez, S. Verdú, and S. Schartz, "Stability properties of slotted Aloha with multipacket reception capability," IEEE Trans. Autom. Control, vol. 33, no. 7, pp. 640-649, Jul. 1988.

[8] H. Takagi and L. Kleinrock, "Optimal transmission ranges for randomly distributed packet radio networks," IEEE Trans. Commun., vol. COM-32, no. 3, pp. 246-257, Mar. 1984.

[9] D. Bertsekas and R. Gallager, Data Networks. Englewood Cliffs, NJ: Prentice-Hall, 1988.

[10] F. Tobagi and L. Kleinrock, "Packet switching in radio channels part II-The hidden terminal," IEEE Trans. Commun., vol. COM-23, no. 12, pp. 1417-1433, Dec. 1975.

[11] P. Karn, "MACA-A new channel access method for packet radio," in Proc. Amateur Radio 9th Computer Networking Conf., London, ON, Canada, Sep. 1990, pp. 134-140.
[12] V. Bhargavan, A. Demers, S. Shenker, and L. Zhang, "MACAW: A media access protocol for wireless LANs," in Proc. ACM SIGCOMM, London, U.K., Aug./Sep. 1994, pp. 212-225.

[13] Talucci and M. Gerla, "MACA-BI (MACA). a wireless MAC," in Proc. IEEE ICUPC, by invitation. San Diego, CA, Oct. 1997, pp. 913-917.

[14] J. Deng and Z. Haas. (1998) Dual Busy Tone Multiple Access (DBTMA): A New Medium Access Control for Packet Radio Networks. [Online]. Available: citeseer.nj.nec.com/deng98dual.html

[15] P. Gupta and P. R. Kumar, "The capacity of wireless networks," IEEE Trans. Inf. Theory, vol. 46, no. 2, pp. 388-404, Mar. 2000.

[16] D. Blough, M. Leoncini, G. Resta, and P. Santi, "On the symmetric range assignment problem in wireless ad hoc networks," in Proc. 2nd IFIP Int. Conf. Theoretical Computer Science (TCS), Montreal, QC, Canada, Aug. 2002, pp. 71-82.

[17] L. M. Kirousis, E. Kranakis, D. Krizanc, and A. Pelc, "Power consumption in packet radio networks," Theor. Comp. Sci., vol. 243, no. 1-2, pp. 289-305, 2000.

[18] M. K. Marina and S. R. Das, "Routing performance in the presence of unidirectional links in multihop wireless networks," in Proc. ACM MOBIHOC, Lausanne, Switzerland, Jun. 2002, pp. 12-23.

[19] D. Blough, M. Leoncini, G. Resta, and P. Santi, "The $k$-neigh protocol for symmetric topology control in ad hoc networks," in Proc. ACM MOBIHOC, Annapolis, MD, Jun. 2003, pp. 141-152.

[20] K. Al Agha and L. Viennot. (2000) On the Efficiency of Multicast. INRIA Tech. Rep. RR-3929. [Online]. Available: http://www.inria.fr/ rrrt/rr-3929.html

[21] J. Lee and L. Miller, CDMA Systems Engineering Handbook. Boston, MA: Artech House, 1998.

[22] F. Baccelli and P. Brémaud, Elements of Queueing Theory, 2nd ed, ser. Applications of Mathematics. Berlin, Germany: Springer-Verlag, 2002.

[23] B. Karp, "Geographic Routing for Wireless Networks," Ph.D. dissertation, Harvard Univ., Cambridge, MA, 2000.

[24] L. Blazevic, J.-Y. L. Boudec, and S. Giordano. A Location Based Routing Method for Irregular Mobile Ad Hoc Networks. [Online]. Available: citeseer.nj.nec.com/blazevic03location.html

[25] P. Jacquet, P. Minet, P. R. Mühlethaler, and N. Rivierre, "Priority and collision detection with active signalling: The channel access mechanism of hiperlan," in Wireless Personnal Commun., Jan. 1997, pp. 11-25.

[26] B. Matern, "Meddelanden fran statens," Skogsforskningsinstitut 49,5, vol. 2, pp. 1-144, 1960.

[27] D. Stoyan, W. Kendall, and J. Mecke, Stochastic Geometry and its Applications. Chichester, U.K.: Wiley, 1995.

[28] P. Mühlethaler and A. Najid, "Optimization of CSMA Multihop Adhoc Network,” INRIA, Rep. RR 4928, 2003.

[29] F. Baccelli and B. Błaszczyszyn, "On a coverage process ranging from the Boolean model to the Poisson-Voronoi tessellation, with applications to wireless communications," Adv. Appl. Probab., vol. 33, pp. 293-323, 2001. 